American Journal of Environmental Sciences 8 (2): 117-129, 2012

ISSN 1553-345X

(C) 2012 Science Publications

\title{
Biomagnification Profiles of Trace Elements through the Food Web of an Integrated Shrimp Mangrove Farm in Ba Ria Vung Tau, South Vietnam
}

\author{
${ }^{1}$ Nguyen Phuc Cam Tu, ${ }^{2}$ Nguyen Ngoc Ha, ${ }^{1}$ Hiroaki Matsuo, \\ ${ }^{3}$ Bui Cach Tuyen, ${ }^{2}$ Shinsuke Tanabe and ${ }^{1}$ Ichiro Takeuchi \\ ${ }^{1}$ Department of Life Environment Conservation, Faculty of Agriculture, \\ Ehime University, Matsuyama, Ehime 790-8566, Japan \\ ${ }^{2}$ Center for Marine Environmental Studies, Ehime University, \\ Matsuyama, Ehime 790-8577, Japan \\ ${ }^{3}$ Research Institute for Biotechnology and Environment, \\ Nong Lam University, Thu Duc District, Hochiminh City, Vietnam
}

\begin{abstract}
Problem statement: Ba Ria Vung Tau (BRVT), a Vietnamese province with one of the fastest growing economies in the country, faces significant environmental challenges, including increased contamination of rivers and estuaries with urban and industrial wastes. Approach: Concentrations of 23 trace elements and stable isotope ratios $\left(\delta^{13} \mathrm{C}\right.$ and $\left.\delta^{15} \mathrm{~N}\right)$ were analyzed in suspended particulate matter, one species of peanut worm, one species of octopus, six species of crustaceans and 13 species of fish collected from an integrated shrimp mangrove farm in the BRVT. Results: Concentrations of $\mathrm{Cu}, \mathrm{Zn}, \mathrm{Se}, \mathrm{Mo}, \mathrm{Ag}, \mathrm{Cd}, \mathrm{Sb}$ and $\mathrm{Bi}$ in octopus, $\mathrm{Mn}, \mathrm{Sr}$ and $\mathrm{Ba}$ in crustaceans and $\mathrm{Hg}$ in fish were the highest among organisms analyzed. Using $\delta^{15} \mathrm{~N}$ values to identify trophic positions, we found that $\mathrm{Zn}, \mathrm{Se}$ and $\mathrm{Hg}$ biomagnified through the food web. The slope for $\mathrm{Hg}$ (0.048) was remarkably lower than those reported for other aquatic food webs. In contrast, the concentrations of most trace elements followed reverse trends (i.e., decreasing concentrations with increasing trophic level). Trace element concentrations we measured in organisms were within safe levels for human consumption according to criteria established by the Ministry of Agriculture, Fisheries and Food (MAFF) in the UK. However, concentrations of $\mathrm{Cu}$ in black tiger shrimps and octopus and $\mathrm{Zn}$ in Commerson's glassy fish were higher than the MAFF guidelines. Conclusion: The results of this study highlight the usefulness of $\delta^{13} \mathrm{C}$ and $\delta^{15} \mathrm{~N}$ as a tool not only for elucidating the trophic position of biota, but also for tracing contaminants within food webs.
\end{abstract}

Key words: Mercury, stable isotope ratios, $\delta^{13} \mathrm{C}, \delta^{15} \mathrm{~N}$, Integrated Shrimp Mangrove Farming Systems (ISMFS), trace elements, food web structure, Ba Ria Vung Tau (BRVT)

\section{INTRODUCTION}

The occurrence of trace elements in aquatic ecosystems of South Vietnam is of concern due to numerous potentially polluting natural and anthropogenic activities. Trace elements have been detected in various biota and environmental samples across this region and there has been concern about sources and concentrations in food items that are frequently consumed by humans and wildlife (Phuong et al., 1998; Anh et al., 2003; Ikemoto et al., 2008; Tu et al., 2008a; 2008b; Agusa et al., 2009). However, little is known about the behaviors of trace elements within Vietnamese estuarine ecosystems, particularly with respect to biomagnification. Biomagnification occurs when concentrations of trace elements increase between two or more trophic levels in a food chain. Among trace elements, As and $\mathrm{Hg}$ show a propensity to biomagnify (Suedel et al., 1994).

Traditional methods of assessing trophic position have depended largely on inferred feeding behavior or stomach content analysis. These methods can be limited, since they usually provide only a "snapshot" of feeding habits for a particular season, life history stage, or location. These snapshots may not necessarily reflect an organism's long-term feeding habits, which can ultimately influence its contaminant load (Atwell et al., 1998). In the last two decades, there has been progress

Corresponding Authors: Nguyen Phuc Cam Tu, Department of Life Environment Conservation, Faculty of Agriculture, Ehime University, Tarumi 3-5-7, Matsuyama City, Ehime 790-8566, Japan Tel/Fax: +81 899469899 
in modeling biomagnification profiles of environmental chemicals and trace elements using stable isotope ratios of bio-elements such as carbon and nitrogen. In general, the nitrogen stable isotope ratio $\left(\delta^{15} \mathrm{~N}\right)$ is about 3-5\%o higher on average in a predator than in its prey (Minagawa and Wada, 1984). Thus, $\delta^{15} \mathrm{~N}$ can be used to estimate the trophic positions of organisms in a food web. In contrast, the carbon stable isotope ratio $\left(\delta^{13} \mathrm{C}\right)$ increases only slightly with increasing trophic level (about $1 \%$ per trophic level). For this reason, $\delta^{13} \mathrm{C}$ is used mostly to identify dietary carbon sources in a food web (DeNiro and Epstein, 1978; Peterson and Fry, 1987). According to Peterson and Fry (1987), these stable isotope analyses complement other methods of studying diets in that the isotopic compositions of tissues are a measure of the assimilated (not just ingested) diet, reflect both long-term and short-term diets in slow and fast turnover tissues and provide a unique way to study food webs of the past, detrital food webs and diets of animals that are hard to observe. Therefore, measurements of trace elements and of $\delta^{15} \mathrm{~N}$ and $\delta^{13} \mathrm{C}$ in the same biota samples enable estimation of biomagnification profiles of trace elements in marine and aquatic food webs. Several studies have used stable isotope analyses to determine trophic position and trophic-level increase in the concentrations of trace elements in marine and coastal food webs (Cabana and Rasmussen, 1994; Kidd et al., 1995; Atwell et al., 1998; Ikemoto et al., 2008). In Vietnam, Ikemoto et al. (2008) reported isotopic trends in the biomagnification of the trace elements $\mathrm{Se}, \mathrm{Rb}$ and $\mathrm{Hg}$ in the Mekong River food web. However, baseline values which represent the primary production level and range of $\delta^{15} \mathrm{~N}$ of a coastal food web may vary from ecosystem to ecosystem, owing to differences in anthropogenic nutrient inputs. The $\delta^{15} \mathrm{~N}$ is reported to increase in areas that are exposed to industrial wastewater, e.g., from food processing factories, the animal husbandry industry and industrial plants (Jones et al., 2001; Costanzo et al., 2004; 2005). Therefore, usage of $\delta^{15} \mathrm{~N}$ for estimating trophic levels requires that all biota samples be collected from the same ecosystem.

Ba Ria Vung Tau (BRVT) Province is located in the southeastern region of Vietnam and is part of the South Key Economic Zone. It encompasses a geographical area of about $2,000 \mathrm{~km}^{2}$ and has a human population of about 800,000 people. The estuarine region of BRVT is an essential nursery and habitat for several commercially important fish and crustacean species. Over the last decade, BRVT has become one of the provinces contributing the largest revenue and economic growth to the state budget. In parallel with the development of industrial enterprise, however,
BRVT is facing significant environmental challenges, such as the contamination of rivers and estuaries with urban and industrial waste and land pollution from similar sources. In particular, the activities of marine shipping, petroleum exploration and exploitation, among others, have progressively expanded and are real threats to the estuarine environment and to sustainable development. Of the contaminants, the major pollutants in the BRVT estuaries are oil and heavy metals (MONRE, 2004).

Integrated Shrimp Mangrove Farming Systems (ISMFS) are one kind of extensive aquaculture that relies on trapping of wild seed during high tides and no provisions are made for supplementary feeding of shrimp. Water exchange and harvesting take place during spring tides as follows: over the duration of a high water tide in daylight hours, a sluice gate is opened to take in water and small shrimp until the pond is full. During night at low water, the water gate is reopened to let pond water flow out. The larger shrimp following the water flow are harvested with a bag net installed at the water gate. Harvesting normally lasts 34 days over each spring tide period in each lunar month and continues throughout the culture period. In addition to the prawn Penaeus spp., which is the main product, other minor species of shrimp, fish and crab are also harvested in the process (Binh et al., 1997).

To determine whether trace element biomagnifications were occurring, we analyzed stable isotopic ratios $\left(\delta^{13} \mathrm{C}\right.$ and $\left.\delta^{15} \mathrm{~N}\right)$ and concentrations of 23 trace elements in organisms and sediments from an ISMFS in the Tan Thanh District, BRVT.

\section{MATERIALS AND METHODS}

Site description and sampling: All samples were collected from a 12 ha ISMFS in the Tan Thanh District, BRVT (10³1.310'N; $\left.107^{\circ} 03.438^{\prime} \mathrm{E}\right)$ (Fig. 1) from 9-11 March, 2007. The study site was located approximately $70 \mathrm{~km}$ southeast of Hochiminh City and about $30 \mathrm{~km}$ northwest of Vung Tau City. The site is adjacent to the Thi Vai River, one of the hotspots of trace element contamination in the country (MONRE, 2004). The pond area includes mangroves, ditches and bare land. The average depth of the water column above the flat was $0.7-1.0 \mathrm{~m}$.

Barramundi seabass Lates calcarifer were caught by angling on 9 and 10 March 2007. On the night of 10 March 2007, we collected the majority of biota samples with a bag net mounted on the water gate, while green crab Scylla serrata was caught using baited trap. 


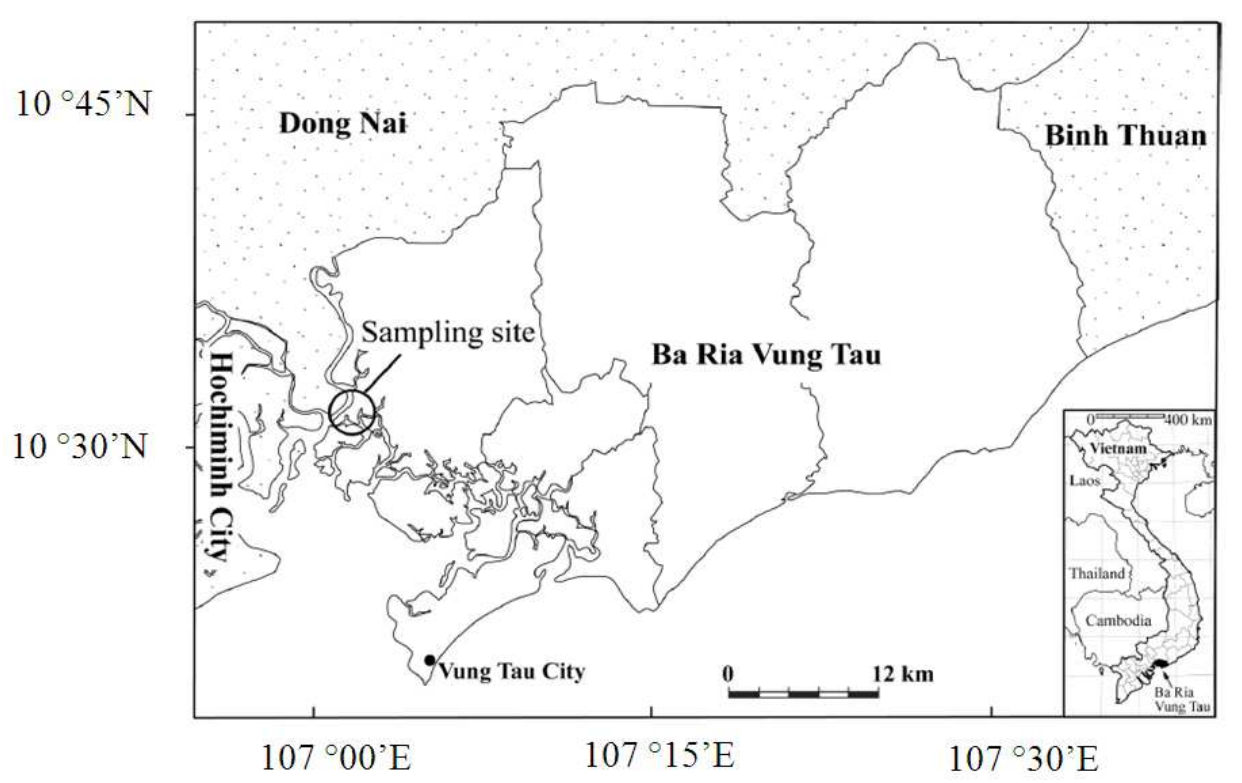

Fig. 1: Map of the study area showing the location of sampling site in an integrated shrimp mangrove farming system, Ba Ria Vung Tau, South Vietnam

On 11 March 2007, a suspended particulate matter (SPM) sample was collected with North Pacific plankton net (NORPAC) $(0.1 \mathrm{~mm}$ mesh size) deployed horizontally at the sluice gate. SPM was washed from the sides of the net into acid-washed plastic bottles with a small volume of pond water. In the laboratory, samples collected with the plankton net were kept in a refrigerator for two to four hours to allow settling. After settling, a brown layer that sank close to the bottom of the bottle was designated SPM. In total, 13 species of fish, six species of crustaceans, one species of worm, one species of octopus and SPM were collected. Two sediment samples were collected using a hand grab taking the top $5 \mathrm{~cm}$ of the sediments. These samples were placed in polyethylene bags, kept in ice, transported to Japan and stored in deep freezer at $<-20^{\circ} \mathrm{C}$ until dissection and chemical analysis. The samples collected for the present study were also used in the analysis of the biomagnification of arsenic species (Tu et al., 2011).

Analysis for stable isotopes: In order to avoid contamination and bias from biota within their digestive tracts, we used muscle tissues of fish, crustaceans, worm and octopus as the standard for stable isotope samples. All samples prepared for $\delta^{13} \mathrm{C}$ and $\delta^{15} \mathrm{~N}$ analysis were rinsed in distilled water, dried in an oven for $24 \mathrm{~h}$ at $60^{\circ} \mathrm{C}$, pulverized to a fine powder and treated with a 2:1 chloroform-methanol solution for 24 $\mathrm{h}$ to remove lipids. Samples were then dried under a fume hood. Sediment and SPM were soaked in $0.1 \mathrm{~N}$
$\mathrm{HCl}$ to remove carbonates, rinsed in distilled water and then dried. Homogenized samples of approximately 1 $\mathrm{mg}$ were loaded into tin cups and $\delta^{13} \mathrm{C}$ and $\delta^{15} \mathrm{~N}$ were measured using a gas chromatograph-combustionisotope ratio mass spectrometer (ANCA-SL, PDZ Europa Ltd., Cheshire, UK). Stable isotope abundances were expressed in the $\delta$ notation as the deviation from standards in parts per thousand (\%o) according to the following equation:

$$
\delta X(\% o)=\left(R_{\text {sample }} / R_{\text {standard }}-1\right) \times 1000
$$

where, $X$ is ${ }^{13} \mathrm{C}$ or ${ }^{15} \mathrm{~N}$ and $R$ is the corresponding ratio ${ }^{13} \mathrm{C} /{ }^{12} \mathrm{C}$ or ${ }^{15} \mathrm{~N} /{ }^{14} \mathrm{~N}$. The $\mathrm{R}_{\text {standard }}$ values were based on PeeDee Belemnite for $\delta^{13} \mathrm{C}$ and atmospheric Nitrogen $\left(\mathrm{N}_{2}\right)$ for $\delta^{15} \mathrm{~N}$. Replicate measurements of the internal laboratory standard (histidine) indicated measurement errors of $\pm 0.1 \%$ or both $\delta^{13} \mathrm{C}$ and $\delta{ }^{15} \mathrm{~N}$ determinations.

Analysis for trace elements: Nine individuals of the peanut worm were pooled after removing their digestive tracts. For other organisms, whole homogenized samples were weighed, deep-frozen and lyophilized for $24 \mathrm{~h}$. They were weighed again to determine their water content and then ground to a fine powder using a mortar and pestle. The average moisture contents were $84.1 \%$ in SPM, $76.2 \%$ in worms, $69.1 \pm 5.1 \%$ in crustaceans, $76.8 \pm 3.5 \%$ in octopus and $70.3 \pm 4.4 \%$ in fish. In order to make comparisons with other studies or guidelines on a wet weight basis, our data 
were converted to wet weight using conversion factors of 3.3 for crustaceans, 4.4 for octopus and 3.4 for fish.

To analyze trace elements, samples of approximately $0.2 \mathrm{~g}$ of freeze-dried specimens were digested with nitric acid. Digestions were carried out in Teflon vessels in a microwave oven (Ethos D, Milestone S.r.l., Sorisole, BG, Italy) under controlled pressure conditions; there were seven digestion steps: 2 , 3, 5, 5, 5 and 10 min under 250, 0, 250, 400, 500 and $400 \mathrm{~W}$, respectively and ventilation for $5 \mathrm{~min}$. The resulting clear solutions were made up to exactly $50 \mathrm{~mL}$ with Milli-Q water. Levels of 22 trace elements (V, Cr, $\mathrm{Mn}, \mathrm{Fe}, \mathrm{Co}, \mathrm{Cu}, \mathrm{Zn}, \mathrm{As}, \mathrm{Se}, \mathrm{Rb}, \mathrm{Sr}, \mathrm{Mo}, \mathrm{Ag}, \mathrm{Cd}$, In, Sn, $\mathrm{Sb}, \mathrm{Cs}, \mathrm{Ba}, \mathrm{Tl}, \mathrm{Pb}$ and $\mathrm{Bi}$ ) were determined with an inductively coupled plasma-mass spectrometer (ICPMS; Agilent 7500cx, Agilent Technologies, Inc., Santa Clara, CA, USA) with yttrium as the internal standard. Mercury was determined using an automated cold vapor analyzer (HG 450, Hiranuma Sangyo Co., Ltd., Mito, Japan). Accuracies of the methods were assessed using certified reference material DOLT3 (National Research Council of Canada) in triplicate and recoveries of all the elements ranged from $90-116 \%$ of the certified values. All data were expressed on a dry weight basis ( $\mu \mathrm{g} / \mathrm{g}$ dry wt.).

Air-dried sediment was sieved through a $0.5 \mathrm{~mm}$ mesh and subsequently pulverized. Sediment analyses were conducted in triplicate. Sediment samples were digested in a mixture of $\mathrm{HNO}_{3}$ and $\mathrm{HF}$ using a microwave system (Ethos D). The microwave digestion programs were set at $2 \mathrm{~min}$ at $250 \mathrm{~W}, 3 \mathrm{~min}$ at $0 \mathrm{~W}, 5$ min at $250 \mathrm{~W}, 5 \mathrm{~min}$ at $400 \mathrm{~W}, 5 \mathrm{~min}$ at $500 \mathrm{~W}, 5 \mathrm{~min}$ at $400 \mathrm{~W}$ and $5 \mathrm{~min}$ for ventilation. To remove $\mathrm{HF}$, digested sediment solution was evaporated by heating. The residue was re-dissolved in $\mathrm{HNO}_{3}$ and then diluted with Milli-Q water. Levels of 19 trace elements (Al, V, $\mathrm{Cr}, \mathrm{Mn}, \mathrm{Fe}, \mathrm{Co}, \mathrm{Cu}, \mathrm{Zn}, \mathrm{As}, \mathrm{Se}, \mathrm{Mo}, \mathrm{Ag}, \mathrm{Cd}, \mathrm{In}, \mathrm{Sn}, \mathrm{Sb}$, $\mathrm{Tl}, \mathrm{Pb}$ and $\mathrm{Bi}$ ) were determined with an ICP-MS (Agilent 7500cx). Rhodium was used as internal standard for correction of matrix effects and instrumental drift in ICP-MS measurements. Mercury was determined using an automated cold vapor analyzer (HG 450). Analytical accuracy was confirmed by analyzing the certified reference material PACS 2 Marine sediment provided by the National Research Council of Canada. The recoveries of trace elements were in the range of 75$123 \%$ of certified values. Concentrations of trace elements in sediment samples are presented on an air dry weight basis ( $\mu \mathrm{g} / \mathrm{g}$ air-dry wt.).

Statistical analyses: Data were statistically analyzed using the statistical package SPSS 15 for Windows (SPSS, Chicago, IL, USA). A Kolmogorov-Smirnov one sample test was conducted to examine goodness of fit to a normal distribution. Most of the variables were not normally distributed and non-parametric tests were used to compare groups. Nonparametric methods were used not only because of non-normal distribution of data, but also because they are most appropriate for small sample sizes. Differences between species were analyzed by the Mann-Whitney U test. As there was no sample replication for the worm and SPM, they were not included in this analysis. To investigate interaction between $\mathrm{Se}$ and $\mathrm{Hg}$, concentrations of $\mathrm{Se}$ and $\mathrm{Hg}$ were converted to molar values (atomic weights 78.96 and $200.59 \mathrm{~g} / \mathrm{mol}$, respectively) to calculate molar ratios. Trace element concentrations were log-transformed to normalize the distribution and simple linear regressions were conducted to verify the existing relationship between $\delta^{15} \mathrm{~N}$ values and trace element concentrations and also, to verify the inter-element relationship between $\mathrm{Se}$ and $\mathrm{Hg}$ (on molar basis). A $p$-value $<0.05$ was considered indicative of statistical significance.

\section{RESULTS}

The biometry and average $\delta^{13} \mathrm{C}$ and $\delta^{15} \mathrm{~N}$ values for all samples are presented in Table 1 . The signature of $\delta^{15} \mathrm{~N}$ for samples ranged from $6.3 \%$ (sediment) to 13.8 $\%$ in swimming crab Charybdis sp. and seabass Lates calcarifer. All species showed a $\delta^{13} \mathrm{C}$ value ranged between $-26.1 \%$ in the SPM to $-13.6 \%$ in the detritivorous fish species Mugil cephalus (Fig. 2).

Concentrations of trace elements in sediments are shown in Table 2. Of the 20 elements analyzed, the concentration of $\mathrm{Fe}$ was the highest (mean, 22,400 $\mu \mathrm{g} / \mathrm{g}$ air-dry wt.), followed by Al $(7,100 \mu \mathrm{g} / \mathrm{g})$, Mn (206 $\mu \mathrm{g} / \mathrm{g}), \mathrm{Zn}(98.8 \mu \mathrm{g} / \mathrm{g}), \mathrm{V}(87 \mu \mathrm{g} / \mathrm{g}), \mathrm{Cr}(60 \mu \mathrm{g} / \mathrm{g})$, $\mathrm{Pb}(20.0 \mu \mathrm{g} / \mathrm{g}), \mathrm{Co}(15 \mu \mathrm{g} / \mathrm{g}), \mathrm{Cu}(14.3 \mu \mathrm{g} / \mathrm{g})$ and $\mathrm{As}$ (14 $\mu \mathrm{g} / \mathrm{g})$; concentrations of Mo $(4.82 \mu \mathrm{g} / \mathrm{g})$ and $\mathrm{Sn}$ $(2.82 \mu \mathrm{g} / \mathrm{g})$ were at moderate levels. The concentrations of Se and toxic elements such as $\mathrm{Ag}, \mathrm{Cd}, \mathrm{In}, \mathrm{Sb}, \mathrm{Hg}, \mathrm{Tl}$ and $\mathrm{Bi}$ were relatively low $(<1 \mu \mathrm{g} / \mathrm{g})$.

The concentrations of trace elements in SPM and whole bodies of crustaceans, fish and octopus are shown in Table 3. The concentrations of several elements such as $\mathrm{V}, \mathrm{Cr}, \mathrm{Fe}, \mathrm{Rb}, \mathrm{Sn}, \mathrm{Ba}, \mathrm{Tl}, \mathrm{Pb}$ and $\mathrm{Bi}$ were relatively high in SPM. The concentrations of $\mathrm{Mn}$, $\mathrm{Co}, \mathrm{Se}, \mathrm{Mo}, \mathrm{In}, \mathrm{Sb}$ and $\mathrm{Cs}$ in the worm, $\mathrm{Cu}, \mathrm{Zn}, \mathrm{As}, \mathrm{Ag}$ and $\mathrm{Cd}$ in octopus, $\mathrm{Sr}$ in crustaceans and $\mathrm{Hg}$ in fish were the highest among organisms analyzed. There were significant differences among crustacean, fish and cephalopod in concentrations of most of trace elements, except for $\mathrm{Fe}, \mathrm{Rb}, \mathrm{In}, \mathrm{Cs}, \mathrm{Tl}$ and $\mathrm{Pb}$ (Mann-Whitney $\mathrm{U}$ test, $p>0.05)$. Concentrations of $\mathrm{Cu}, \mathrm{Zn}, \mathrm{Se}, \mathrm{Mo} \mathrm{Ag}, \mathrm{Cd}$, $\mathrm{Sb}$ and $\mathrm{Bi}$ in octopus were significantly higher than those in the other two phyla $(p<0.05)$. 
Am. J. Environ. Sci., 8 (2): 117-129, 2012

Table 1: Sample details and stable isotope ratios in the integrated shrimp mangrove farming system, Ba Ria Vung Tau, South Vietnam

\begin{tabular}{|c|c|c|c|c|c|c|c|c|c|c|}
\hline \multirow[b]{2}{*}{ Species } & \multirow[b]{2}{*}{ Scientific name } & \multirow[b]{2}{*}{ ID } & \multirow[b]{2}{*}{$n^{a}$} & \multirow[b]{2}{*}{$n^{b}$} & \multicolumn{2}{|c|}{ Body size $^{\mathrm{c}}(\mathrm{mm})$} & \multicolumn{2}{|c|}{ Body weight (g) } & \multirow{2}{*}{$\begin{array}{l}\delta^{15} \mathrm{~N}(\%) \\
\text { Mean } \pm \text { SD }\end{array}$} & \multirow{2}{*}{$\begin{array}{l}\delta^{13} \mathrm{C}(\% o) \\
\text { Mean } \pm \text { SD }\end{array}$} \\
\hline & & & & & Mean $\pm \mathrm{SD}^{\mathrm{d}}$ & Range & Mean \pm SD & Range & & \\
\hline Sediment & & & 4 & 2 & - & - & - & - & $6.3 \pm 0.3$ & $-25.4 \pm 0.0$ \\
\hline $\begin{array}{l}\text { Peanut worm } \\
\text { Crustacean }\end{array}$ & Phascolosoma arcuatum & PW & 6 & 1 & - & - & $6.1 \pm 1.9$ & $(3.3-10.2)$ & $10.2 \pm 0.5$ & $-20.1 \pm 1.1$ \\
\hline Box crab & Calappa sp. & $\mathrm{C} 1$ & 6 & 3 & $40.6 \pm 10.9$ & $(28.3-49.1)$ & $65.9 \pm 41.4$ & $(20.8-102.1)$ & $11.8 \pm 0.5$ & $-21.1 \pm 0.3$ \\
\hline Gazami crab & Portunus trituberculatus & $\mathrm{C} 2$ & 6 & 3 & $39.7 \pm 8.2$ & $(33.0-48.8)$ & $44.2 \pm 33.4$ & $(21.0-82.5)$ & $9.2 \pm 0.3$ & $-14.5 \pm 0.2$ \\
\hline Green crab & Scylla serrata & $\mathrm{C} 3$ & 6 & 1 & 43.6 & - & 45.9 & - & $9.8 \pm 0.1$ & $-20.2 \pm 0.3$ \\
\hline Swimming crab & Charybdis sp. & $\mathrm{C} 4$ & 5 & 5 & $34.4 \pm 3.2$ & $(31.4-39.4)$ & $25.7 \pm 10.7$ & $(17.0-43.2)$ & $13.8 \pm 1.0$ & $-20.3 \pm 0.7$ \\
\hline Black tiger shrimp & Penaeus monodon & S1 & 6 & 3 & $19.4 \pm 2.7$ & $(16.3-21.0)$ & $42.3 \pm 13.1$ & $(31.2-56.8)$ & $11.8 \pm 1.0$ & $-19.9 \pm 3.0$ \\
\hline $\begin{array}{l}\text { Banana shrimp } \\
\text { Cephalopod }\end{array}$ & Penaeus merguiensis & $\mathrm{S} 2$ & 7 & 4 & $10.5 \pm 1.1$ & $(8.1-12.0)$ & $8.8 \pm 2.3$ & $(4.0-11.7)$ & $10.0 \pm 0.8$ & $-15.8 \pm 2.4$ \\
\hline $\begin{array}{l}\text { Octopus } \\
\text { Fish }\end{array}$ & Octopus sp. & $\mathrm{O}$ & 6 & 3 & - & - & $27.4 \pm 15.0$ & $(14.8-44.0)$ & $12.0 \pm 1.3$ & $-18.8 \pm 1.7$ \\
\hline Spotted goby & Acanthogobius flavimanus & F1 & 6 & 5 & - & - & $10.3 \pm 4.7$ & $(4.1-18.4)$ & $13.6 \pm 0.2$ & $-18.3 \pm 0.3$ \\
\hline Duckbill sleeper & Butis butis & $\mathrm{F} 2$ & 6 & 1 & 17.2 & - & 25.6 & - & $13.5 \pm 1.0$ & $-17.2 \pm 1.5$ \\
\hline Tufted sole & Dexillichthys muelleri & F3 & 6 & 1 & 22.5 & - & 270.6 & - & $11.0 \pm 0.2$ & $-19.9 \pm 0.1$ \\
\hline Golden rabbit fish & Siganus sp. & $\mathrm{F} 4$ & 6 & 2 & 20.5 & $(18.0-23.0)$ & 90.1 & $(72.4-107.7)$ & $13.0 \pm 0.2$ & $-20.1 \pm 1.7$ \\
\hline Mullet & Mugil cephalus & F5 & 6 & 1 & 21.3 & - & 126.3 & - & $9.6 \pm 0.2$ & $-13.6 \pm 0.1$ \\
\hline Grey eel catfish & Plotosus canius & F6 & 6 & 2 & 35.1 & $(30.4-39.7)$ & 197.6 & $(113.7-281.4)$ & $13.6 \pm 0.2$ & $-18.2 \pm 0.1$ \\
\hline Therapon & Therapon sp. & F7 & 6 & 2 & 18.8 & $(18.1-19.4)$ & 103.5 & $(98.3-108.7)$ & $13.0 \pm 0.1$ & $-16.4 \pm 0.2$ \\
\hline Sillago & Sillago sihama & F8 & 6 & 4 & - & - & $10.4 \pm 4.3$ & $(2.6-18.3)$ & $13.2 \pm 0.4$ & $-16.2 \pm 0.5$ \\
\hline Tilapia & Oreochromis niloticus & F9 & 6 & 4 & $16.0 \pm 1.3$ & $(14.4-17.5)$ & $77.9 \pm 16.8$ & $(53.5-98.4)$ & $13.1 \pm 0.6$ & $-18.6 \pm 1.6$ \\
\hline Spotted scat & Scatophagus argus & F10 & 6 & 4 & $11.8 \pm 2.2$ & $(9.7-14.5)$ & $59.1 \pm 33.5$ & $(27.5-103.3)$ & $11.0 \pm 1.0$ & $-15.7 \pm 0.7$ \\
\hline Commerson's glassy & y Ambassis commersoni & F11 & 6 & 2 & 9.7 & $(7.1-12.2)$ & 12.3 & $(5.1-20.2)$ & $13.2 \pm 0.6$ & $-16.5 \pm 0.8$ \\
\hline Shortnose ponyfish & Leiognathus brevirostris & F12 & 6 & 1 & $9.3 \pm 0.4$ & $(8.7-9.8)$ & $13.6 \pm 1.1$ & $(12.4-15.6)$ & $13.4 \pm 0.7$ & $-17.5 \pm 1.7$ \\
\hline Barramundi seabass & Lates calcarifer & F13 & 6 & 3 & $45.1 \pm 16.3$ & $(28.0-60.4)$ & $1693 \pm 1410$ & $(309-3128)$ & $13.8 \pm 0.3$ & $-18.1 \pm 2.1$ \\
\hline
\end{tabular}

Table 2: Trace element concentrations (mean \pm standard deviation, $\mu \mathrm{g} / \mathrm{g}$ air-dry wt.) in sediments from the integrated shrimp mangrove farming system, Ba Ria Vung Tau, South Vietnam

\begin{tabular}{lll}
\hline Element & Sediment 1 & Sediment 2 \\
\hline $\mathrm{Al}$ & $6120 \pm 225$ & $8080 \pm 638$ \\
$\mathrm{~V}$ & $87 \pm 1$ & $87 \pm 2$ \\
$\mathrm{Cr}$ & $60 \pm 2$ & $59 \pm 1$ \\
$\mathrm{Mn}$ & $210 \pm 3$ & $201 \pm 6$ \\
$\mathrm{Fe}$ & $22200 \pm 734$ & $22700 \pm 366$ \\
$\mathrm{Co}$ & $14 \pm 0$ & $16 \pm 0$ \\
$\mathrm{Cu}$ & $14.3 \pm 0.2$ & $14.3 \pm 0.2$ \\
$\mathrm{Zn}$ & $96.5 \pm 0.1$ & $101 \pm 2$ \\
$\mathrm{As}$ & $14 \pm 0$ & $14 \pm 0$ \\
$\mathrm{Se}$ & $0.46 \pm 0.02$ & $0.44 \pm 0.01$ \\
$\mathrm{Mo}$ & $4.76 \pm 0.08$ & $4.88 \pm 0.07$ \\
$\mathrm{Ag}$ & $0.081 \pm 0.001$ & $0.080 \pm 0.002$ \\
$\mathrm{Cd}$ & $0.148 \pm 0.006$ & $0.146 \pm 0.002$ \\
$\mathrm{In}$ & $0.060 \pm 0.000$ & $0.059 \pm 0.001$ \\
$\mathrm{Sn}$ & $2.81 \pm 0.07$ & $2.82 \pm 0.07$ \\
$\mathrm{Sb}$ & $0.766 \pm 0.008$ & $0.762 \pm 0.007$ \\
$\mathrm{Hg}$ & $0.035 \pm 0.002$ & $0.034 \pm 0.004$ \\
$\mathrm{Tl}$ & $0.486 \pm 0.005$ & $0.506 \pm 0.004$ \\
$\mathrm{~Pb}$ & $19.6 \pm 0.4$ & $20.3 \pm 0.3$ \\
$\mathrm{Bi}$ & $0.320 \pm 0.007$ & $0.328 \pm 0.007$ \\
\hline
\end{tabular}

Crustaceans had the highest concentrations of $\mathrm{Mn}, \mathrm{Sr}$ and $\mathrm{Ba}$, whereas the cephalopod had the lowest $(p<0.05)$. Concentrations of $\mathrm{Co}$, as and $\mathrm{Sb}$ in crustaceans and octopus were higher than those in fish $(p<0.05)$. Chromium, $\mathrm{Cs}$ and $\mathrm{Hg}$ levels in fish were greater than those in crustaceans $(p<0.05)$, although neither phylum was significantly different from octopus.

Relationships between $\delta^{15} \mathrm{~N}$ signatures and logtransformed concentrations of trace elements were examined to investigate trophic level-dependent accumulation of trace elements in the food web (Table 4). In the ISMFS food web, $\log$ concentrations of $\mathrm{Zn}$, $\mathrm{Se}$ and $\mathrm{Hg}$ in whole body samples significantly increased (Simple linear regression, $p<0.05$ ) with increasing $\delta^{15} \mathrm{~N}$ (Table 4 and Fig. 3). The slopes for these elements on dry weight basis were 0.040 for logtransformed $\mathrm{Zn}, 0.029$ for log-transformed Se and 0.048 for log-transformed $\mathrm{Hg}$ (Table 4 and Fig. 3).

Significant negative slopes on dry weight basis $(p<0.05)$ were observed for log-transformed V (-0.085), Fe (-0.115), Co (-0.146), Cu (-0.152), Rb (-0.033), Mo (-0.114), Ag (-0.164), Cd (-0.128), Sn (-0.075), Cs ($0.041), \mathrm{Ba}(-0.090), \mathrm{Tl}(-0.117), \mathrm{Pb}(-0.095)$ and $\mathrm{Bi}(-$ 0.085) (Table 4, Fig. 3). Trophic level-dependent accumulations of $\mathrm{Cr}, \mathrm{Mn}, \mathrm{As}, \mathrm{Sr}$, in and $\mathrm{Sb}$ were not found in these organisms $(p>0.05)$.

Molar ratios of $\mathrm{Se}: \mathrm{Hg}$ varied markedly between individual and species in organisms from this ISMFS (Table 5). Peanut worm presented the highest Se:Hg molar concentration ratio (288), whereas molar ratio in SPM was the lowest (24) (Table 5). The Se: Hg molar ratio ranged from 52-141 and from 36-227 in crustaceans and fishes, respectively and 105 in octopus. 
Am. J. Environ. Sci., 8 (2): 117-129, 2012

Table 3: Trace element concentrations (mean \pm standard deviation, $\mu \mathrm{g} / \mathrm{g}$ dry wt.) in whole organisms from the integrated shrimp mangrove farming system, Ba Ria Vung Tau, South Vietnam. Values in parentheses indicate range. See Table 1 for full generic names abbreviated here

\begin{tabular}{|c|c|c|c|c|c|c|c|c|c|c|c|c|c|}
\hline Species & Moisture (\%) & $\mathrm{v}$ & $\mathrm{Cr}$ & $\mathrm{Mn}$ & $\mathrm{Fe}$ & Co & $\mathrm{Cu}$ & $\mathrm{Zn}$ & As & Se & $\mathrm{Rb}$ & $\mathrm{Sr}$ & Mo \\
\hline SPM & 84.1 & 11 & 18 & 150 & 10100 & 4.3 & 4.63 & 30.1 & 5.6 & 0.63 & 7.05 & 71.6 & 3.83 \\
\hline \multicolumn{14}{|l|}{ Sipuncula } \\
\hline Phascolosoma arcuatum & 76.2 & 7.1 & 4.4 & 605 & 4780 & 11 & 4.87 & 53.2 & 7.4 & 5.2 & 3.50 & 29.8 & 4.87 \\
\hline \multicolumn{14}{|l|}{ Crustacean } \\
\hline Calappa sp. & 61.4 & $\begin{array}{l}0.70 \pm 0.26 \\
(0.42-0.95)\end{array}$ & $\begin{array}{l}1.8 \pm 1.0 \\
(0.87-2.9)\end{array}$ & $\begin{array}{l}371 \pm 201 \\
(240-603)\end{array}$ & $\begin{array}{l}304 \pm 123 \\
(168-407)\end{array}$ & $\begin{array}{l}1.1 \pm 0.1 \\
(1.1-1.2)\end{array}$ & $\begin{array}{l}20.9 \pm 3.3 \\
(18.7-24.7)\end{array}$ & $\begin{array}{l}56.6 \pm 8.2 \\
(47.6-63.8)\end{array}$ & $\begin{array}{l}4.8 \pm 0.6 \\
(4.1-5.3)\end{array}$ & $\begin{array}{l}0.73 \pm 0.14 \\
(0.62-0.89)\end{array}$ & $\begin{array}{l}1.95 \pm 0.61 \\
(1.31-2.52)\end{array}$ & $\begin{array}{l}3157 \pm 108 \\
(3055-3271)\end{array}$ & $\begin{array}{l}0.307 \pm 0.140 \\
(0.202-0.466)\end{array}$ \\
\hline Portunus trituberculatus & 68.8 & $\begin{array}{l}0.32 \pm 0.05 \\
(0.28-0.38)\end{array}$ & $\begin{array}{l}0.98 \pm 1.3 \\
(0.20-2.5)\end{array}$ & $\begin{array}{l}71.4 \pm 21.2 \\
(55.9-95.6)\end{array}$ & $\begin{array}{l}162 \pm 28 \\
(130-183)\end{array}$ & $\begin{array}{l}1.1 \pm 0.5 \\
(0.58-1.5)\end{array}$ & $\begin{array}{l}38.4 \pm 3.2 \\
(34.8-40.9)\end{array}$ & $\begin{array}{l}41.7 \pm 10.2 \\
(33.7-53.1)\end{array}$ & $\begin{array}{l}1.8 \pm 0.4 \\
(1.3-2.2)\end{array}$ & $\begin{array}{l}0.65 \pm 0.15 \\
(0.48-0.75)\end{array}$ & $\begin{array}{l}2.34 \pm 0.25 \\
(2.06-2.52)\end{array}$ & $\begin{array}{l}2606 \pm 370 \\
(2243-2983)\end{array}$ & $\begin{array}{l}0.295 \pm 0.177 \\
(0.148-0.491)\end{array}$ \\
\hline Scylla serrata & 72.2 & 0.42 & 0.32 & 108 & 225 & 0.63 & 31.8 & 50.8 & 3.6 & 1.0 & 2.25 & 2973 & 0.244 \\
\hline Charybdis sp. & 68.4 & $\begin{array}{l}0.39 \pm 0.12 \\
(0.25-0.58)\end{array}$ & $\begin{array}{l}0.46 \pm 0.17 \\
(0.25-0.66)\end{array}$ & $\begin{array}{l}435 \pm 246 \\
(109-662)\end{array}$ & $\begin{array}{l}166 \pm 44 \\
(109-224)\end{array}$ & $\begin{array}{l}0.84 \pm 0.23 \\
(0.49-1.1)\end{array}$ & $\begin{array}{l}29.7 \pm 10.9 \\
(14.8-43.2)\end{array}$ & $\begin{array}{l}57.6 \pm 13.4 \\
(45.4-78.7)\end{array}$ & $\begin{array}{l}7.8 \pm 1.7 \\
(6.5-11)\end{array}$ & $\begin{array}{l}1.2 \pm 0.3 \\
(0.81-1.5)\end{array}$ & $\begin{array}{l}2.46 \pm 0.16 \\
(2.30-2.66)\end{array}$ & $\begin{array}{l}2760 \pm 110 \\
(2626-2892)\end{array}$ & $\begin{array}{l}0.245 \pm 0.097 \\
(0.128-0.341)\end{array}$ \\
\hline Penaeus monodon & 71.5 & $\begin{array}{l}0.30 \pm 0.04 \\
(0.28-0.35)\end{array}$ & $\begin{array}{l}3.9 \pm 1.8 \\
(2.4-5.9)\end{array}$ & $\begin{array}{l}16.8 \pm 8.7 \\
(9.23-26.3)\end{array}$ & $\begin{array}{l}155 \pm 25 \\
(133-182)\end{array}$ & $\begin{array}{l}0.62 \pm 0.26 \\
(0.34-0.86)\end{array}$ & $\begin{array}{l}90.5 \pm 14.0 \\
(77.0-105)\end{array}$ & $\begin{array}{l}82.7 \pm 9.5 \\
(71.8-89)\end{array}$ & $\begin{array}{l}11 \pm 8 \\
(5.5-20)\end{array}$ & $\begin{array}{l}1.5 \pm 0.2 \\
(1.3-1.7)\end{array}$ & $\begin{array}{l}3.91 \pm 0.20 \\
(3.74-4.13)\end{array}$ & $\begin{array}{l}748 \pm 105 \\
(652-860)\end{array}$ & $\begin{array}{l}0.464 \pm 0.103 \\
(0.363-0.569)\end{array}$ \\
\hline Penaeus merguiensis & 73.6 & $\begin{array}{l}0.46 \pm 0.08 \\
(0.37-0.55)\end{array}$ & $\begin{array}{l}2.6 \pm 1.5 \\
(1.1-4.1)\end{array}$ & $\begin{array}{l}9.06 \pm 2.00 \\
(6.06-10.1)\end{array}$ & $\begin{array}{l}212 \pm 23 \\
(191-242)\end{array}$ & $\begin{array}{l}0.50 \pm 0.10 \\
(0.37-0.62)\end{array}$ & $\begin{array}{l}43.5 \pm 0.9 \\
(42.6-44.4)\end{array}$ & $\begin{array}{l}62.4 \pm 6.3 \\
(55.8-69.7)\end{array}$ & $\begin{array}{l}3.0 \pm 1.5 \\
(1.9-5.1)\end{array}$ & $\begin{array}{l}1.3 \pm 0.6 \\
(0.88-2.1)\end{array}$ & $\begin{array}{l}4.23 \pm 0.64 \\
(3.28-4.59)\end{array}$ & $\begin{array}{l}678 \pm 121 \\
(506-770)\end{array}$ & $\begin{array}{l}0.228 \pm 0.068 \\
(0.142-0.308)\end{array}$ \\
\hline \multicolumn{14}{|l|}{ Cephalopod } \\
\hline Octopus sp. & 76.8 & $\begin{array}{l}0.43 \pm 0.37 \\
(0.19-0.86)\end{array}$ & $\begin{array}{l}2.0 \pm 1.3 \\
(0.86-3.4)\end{array}$ & $\begin{array}{l}4.42 \pm 0.58 \\
(4.03-5.09)\end{array}$ & $\begin{array}{l}281 \pm 234 \\
(120-550)\end{array}$ & $\begin{array}{l}1.2 \pm 0.3 \\
(1.0-1.6)\end{array}$ & $\begin{array}{l}107 \pm 23 \\
(81.9-126)\end{array}$ & $\begin{array}{l}179 \pm 37 \\
(136-205)\end{array}$ & $\begin{array}{l}8.5 \pm 3.5 \\
(6.3-13)\end{array}$ & $\begin{array}{l}2.0 \pm 0.4 \\
(1.6-2.4)\end{array}$ & $\begin{array}{l}3.60 \pm 0.29 \\
(3.27-3.81)\end{array}$ & $\begin{array}{l}14.6 \pm 5.1 \\
(10.2-20.2)\end{array}$ & $\begin{array}{l}0.600 \pm 0.199 \\
(0.371-0.739)\end{array}$ \\
\hline \multicolumn{14}{|l|}{ Fish } \\
\hline Acanthogobius flavimanus & 73.7 & $\begin{array}{l}0.69 \pm 0.10 \\
(0.57-0.85)\end{array}$ & $\begin{array}{l}2.2 \pm 0.7 \\
(1.4-3.2)\end{array}$ & $\begin{array}{l}20.7 \pm 12.2 \\
(14.8-42.5)\end{array}$ & $\begin{array}{l}119 \pm 44 \\
(65.8-166)\end{array}$ & $\begin{array}{l}0.40 \pm 0.05 \\
(0.37-0.48)\end{array}$ & $\begin{array}{l}1.21 \pm 0.32 \\
(0.784-1.58)\end{array}$ & $\begin{array}{l}81.4 \pm 23.3 \\
(50.1-107)\end{array}$ & $\begin{array}{l}1.4 \pm 0.2 \\
(1.0-1.6)\end{array}$ & $\begin{array}{l}1.1 \pm 0.3 \\
(0.77-1.5)\end{array}$ & $\begin{array}{l}2.73 \pm 0.26 \\
(2.46-3.06)\end{array}$ & $\begin{array}{l}320 \pm 112 \\
(191-486)\end{array}$ & $\begin{array}{l}0.098 \pm 0.025 \\
(0.073-0.125)\end{array}$ \\
\hline Butis butis & 71.7 & 0.074 & 1.8 & 7.04 & 43.4 & 0.053 & 1.02 & 72.9 & 2.0 & 1.2 & 2.38 & 213 & 0.047 \\
\hline Dexillichthys muelleri & 74.9 & 1.5 & 1.6 & 9.37 & 316 & 1.5 & 1.93 & 65 & 4.3 & 2.8 & 3.36 & 179 & 0.129 \\
\hline Siganus sp. & 67.7 & $\begin{array}{l}0.69 \\
(0.21-1.2)\end{array}$ & $\begin{array}{l}4.4 \\
(1.9-6.9)\end{array}$ & $\begin{array}{l}30.3 \\
(4.32-56.2)\end{array}$ & $\begin{array}{l}126 \\
(68.5-184)\end{array}$ & $\begin{array}{l}0.69 \\
(0.052-1.3)\end{array}$ & $\begin{array}{l}0.947 \\
(0.697-1.20)\end{array}$ & $\begin{array}{l}45.4 \\
(43.6-47.1)\end{array}$ & $\begin{array}{l}3.4 \\
(1.9-5.0)\end{array}$ & $\begin{array}{l}1.5 \\
(1.4-1.5)\end{array}$ & $\begin{array}{l}2.42 \\
(2.21-2.63)\end{array}$ & $\begin{array}{l}159 \\
(112-205)\end{array}$ & $\begin{array}{l}0.120 \\
(0.103-0.136)\end{array}$ \\
\hline Mugil cephalus & 62.8 & 4.5 & 2.9 & 4.05 & 363 & 1.1 & 0.894 & 40.1 & 1.7 & 0.58 & 2.45 & 140 & 0.130 \\
\hline Plotosus canius & 72.5 & $\begin{array}{l}0.57 \\
(0.38-0.76)\end{array}$ & $\begin{array}{l}8.3 \\
(4.5-12)\end{array}$ & $\begin{array}{l}5.38 \\
(4.25-6.52)\end{array}$ & $\begin{array}{l}286 \\
(132-439)\end{array}$ & $\begin{array}{l}0.18 \\
(0.083-0.28)\end{array}$ & $\begin{array}{l}1.67 \\
(1.62-1.73)\end{array}$ & $\begin{array}{l}48.1 \\
(44.5-51.7)\end{array}$ & $\begin{array}{l}4.8 \\
(3.9-5.7)\end{array}$ & $\begin{array}{l}1.6 \\
(1.5-1.6)\end{array}$ & $\begin{array}{l}4.24 \\
(3.77-4.70)\end{array}$ & $\begin{array}{l}288 \\
(251-325)\end{array}$ & $\begin{array}{l}0.246 \\
(0.153-0.339)\end{array}$ \\
\hline Therapon sp. & 65.0 & $\begin{array}{l}0.14 \\
(0.11-0.16)\end{array}$ & $\begin{array}{l}5.0 \\
(4.4-5.7)\end{array}$ & $\begin{array}{l}3.49 \\
(3.03-3.95)\end{array}$ & $\begin{array}{l}80.2 \\
(77.5-82.9)\end{array}$ & $\begin{array}{l}0.073 \\
(0.073-0.074)\end{array}$ & $\begin{array}{l}1.25 \\
(1.19-1.30)\end{array}$ & $\begin{array}{l}100 \\
(91.5-109)\end{array}$ & $\begin{array}{l}0.87 \\
(0.80-0.94)\end{array}$ & $\begin{array}{l}1.0 \\
(0.97-1.1)\end{array}$ & $\begin{array}{l}1.68 \\
(1.52-1.83)\end{array}$ & $\begin{array}{l}168 \\
(137-200)\end{array}$ & $\begin{array}{l}0.090 \\
(0.086-0.094)\end{array}$ \\
\hline Sillago sihama & 75.8 & $\begin{array}{l}0.76 \pm 0.14 \\
(0.65-1.0)\end{array}$ & $\begin{array}{l}13 \pm 20 \\
(1.7-43)\end{array}$ & $\begin{array}{l}7.26 \pm 1.07 \\
(6.63-8.86)\end{array}$ & $\begin{array}{l}211 \pm 51 \\
(172-286)\end{array}$ & $\begin{array}{l}0.22 \pm 0.12 \\
(0.15-0.40)\end{array}$ & $\begin{array}{l}2.24 \pm 1.17 \\
(1.20-3.62)\end{array}$ & $\begin{array}{l}86.1 \pm 3.7 \\
(81-89.1)\end{array}$ & $\begin{array}{l}2.4 \pm 0.6 \\
(1.6-3.0)\end{array}$ & $\begin{array}{l}1.2 \pm 0.1 \\
(1.1-1.4)\end{array}$ & $\begin{array}{l}2.26 \pm 0.07 \\
(2.15-2.31)\end{array}$ & $\begin{array}{l}294 \pm 55 \\
(241-370)\end{array}$ & $\begin{array}{l}0.134 \pm 0.059 \\
(0.076-0.214)\end{array}$ \\
\hline Oreochromis niloticus & 68.3 & $\begin{array}{l}1.2 \pm 0.7 \\
(0.6-2.1)\end{array}$ & $\begin{array}{l}13 \pm 8 \\
(3.9-21)\end{array}$ & $\begin{array}{l}51.1 \pm 12.0 \\
(35.7-60.8)\end{array}$ & $\begin{array}{l}439 \pm 225 \\
(186-721)\end{array}$ & $\begin{array}{l}0.52 \pm 0.06 \\
(0.45-0.59)\end{array}$ & $\begin{array}{l}1.60 \pm 0.12 \\
(1.45-1.74)\end{array}$ & $\begin{array}{l}100 \pm 3 \\
(96.8-103)\end{array}$ & $\begin{array}{l}3.6 \pm 1.0 \\
(2.6-5.0)\end{array}$ & $\begin{array}{l}1.9 \pm 0.3 \\
(1.5-2.2)\end{array}$ & $\begin{array}{l}2.31 \pm 0.42 \\
(1.96-2.81)\end{array}$ & $\begin{array}{l}313 \pm 50 \\
(253-368)\end{array}$ & $\begin{array}{l}0.263 \pm 0.121 \\
(0.116-0.382)\end{array}$ \\
\hline Scatophagus argus & 64.7 & $\begin{array}{l}1.1 \pm 0.3 \\
(0.82-1.6)\end{array}$ & $\begin{array}{l}8.2 \pm 4.1 \\
(5.1-14)\end{array}$ & $\begin{array}{l}15.8 \pm 10.8 \\
(9.12-31.9)\end{array}$ & $\begin{array}{l}423 \pm 243 \\
(166-751)\end{array}$ & $\begin{array}{l}0.60 \pm 0.13 \\
(0.43-0.72)\end{array}$ & $\begin{array}{l}1.67 \pm 0.13 \\
(1.53-1.84)\end{array}$ & $\begin{array}{l}52 \pm 6.5 \\
(42.5-57.2)\end{array}$ & $\begin{array}{l}1.4 \pm 0.4 \\
(1.0-1.9)\end{array}$ & $\begin{array}{l}0.79 \pm 0.14 \\
(0.59-0.92)\end{array}$ & $\begin{array}{l}3.17 \pm 0.60 \\
(2.68-3.97)\end{array}$ & $\begin{array}{l}116 \pm 16 \\
(97-136)\end{array}$ & $\begin{array}{l}0.190 \pm 0.060 \\
(0.132-0.273)\end{array}$ \\
\hline Ambassis commersoni & 74.6 & $\begin{array}{l}0.61 \\
(0.58-0.64)\end{array}$ & $\begin{array}{l}14 \\
(8.2-20)\end{array}$ & $\begin{array}{l}18.2 \\
(16.3-20.1)\end{array}$ & $\begin{array}{l}213 \\
(210-217)\end{array}$ & $\begin{array}{l}0.20 \\
(0.19-0.22)\end{array}$ & $\begin{array}{l}1.43 \\
(1.40-1.46)\end{array}$ & $\begin{array}{l}187 \\
(153-221)\end{array}$ & $\begin{array}{l}1.4 \\
(1.1-1.7)\end{array}$ & $\begin{array}{l}1.7 \\
(1.6-1.7)\end{array}$ & $\begin{array}{l}2.26 \\
(2.21-2.30)\end{array}$ & $\begin{array}{l}347 \\
(329-365)\end{array}$ & $\begin{array}{l}0.212 \\
(0.132-0.293)\end{array}$ \\
\hline Leiognathus brevirostris & 67.4 & 0.64 & 3.0 & 8.94 & 387 & 0.21 & 1.93 & 106 & 1.7 & 1.0 & 1.88 & 168 & 0.083 \\
\hline Lates calcarifer & 70.2 & $\begin{array}{l}0.15 \pm 0.1 \\
(0.049-0.24)\end{array}$ & $\begin{array}{l}2.3 \pm 1.2 \\
(1.2-3.7)\end{array}$ & $\begin{array}{l}1.73 \pm 0.55 \\
(1.31-2.36)\end{array}$ & $\begin{array}{l}51.8 \pm 10.6 \\
(42.9-63.5)\end{array}$ & $\begin{array}{l}0.031 \pm 0.004 \\
(0.028-0.036)\end{array}$ & $\begin{array}{l}1.89 \pm 1.16 \\
(1.10-3.22)\end{array}$ & $\begin{array}{l}47 \pm 2.2 \\
(45.1-49.5)\end{array}$ & $\begin{array}{l}1.6 \pm 0.3 \\
(1.3-1.8)\end{array}$ & $\begin{array}{l}1.0 \pm 0.2 \\
(0.88-1.2)\end{array}$ & $\begin{array}{l}2.06 \pm 0.49 \\
(1.54-2.50)\end{array}$ & $\begin{array}{l}274 \pm 68 \\
(223-351)\end{array}$ & $\begin{array}{l}0.026 \pm 0.007 \\
(0.018-0.031)\end{array}$ \\
\hline
\end{tabular}

Table 3: Continue

\begin{tabular}{|c|c|c|c|c|c|c|c|c|c|c|c|}
\hline $\begin{array}{l}\text { Species } \\
\end{array}$ & $\mathrm{Ag}$ & $\mathrm{Cd}$ & In & Sn & $\mathrm{Sb}$ & $\mathrm{Cs}$ & $\mathrm{Ba}$ & $\mathrm{Hg}$ & $\mathrm{Tl}$ & $\mathrm{Pb}$ & $\mathrm{Bi}$ \\
\hline SPM & 0.020 & 0.037 & 0.011 & 0.381 & 0.03 & 0.69 & 12 & 0.067 & 0.043 & 6.25 & 0.109 \\
\hline \multicolumn{12}{|l|}{ Sipuncula } \\
\hline \multicolumn{10}{|l|}{ Crustacean } & 4.58 & 0.032 \\
\hline Calappa sp. & $\begin{array}{l}0.090 \pm 0.050 \\
(0.049-0.15)\end{array}$ & $\begin{array}{l}0.017 \pm 0.005 \\
(0.014-0.023)\end{array}$ & $\begin{array}{l}0.001 \pm 0.001 \\
(0.001-0.002)\end{array}$ & $\begin{array}{l}0.096 \pm 0.012 \\
(0.086-0.109)\end{array}$ & $\begin{array}{l}0.02 \pm 0.01 \\
(0.02-0.04)\end{array}$ & $\begin{array}{l}0.05 \pm 0.02 \\
(0.03-0.07)\end{array}$ & $\begin{array}{l}10 \pm 0.0 \\
(9.5-10)\end{array}$ & $\begin{array}{l}0.025 \pm 0.01 \\
(0.020-0.033)\end{array}$ & $\begin{array}{l}0.003 \pm 0.000 \\
(0.003-0.004)\end{array}$ & $\begin{array}{l}0.640 \pm 0.593 \\
(0.156-1.30)\end{array}$ & $\begin{array}{l}0.002 \pm 0.001 \\
(0.001-0.004)\end{array}$ \\
\hline Portunus trituberculatus & $\begin{array}{l}0.10 \pm 0.02 \\
(0.080-0.12)\end{array}$ & $\begin{array}{l}0.031 \pm 0.008 \\
(0.023-0.036)\end{array}$ & $\begin{array}{l}<0.001 \\
(<0.001-0.001)\end{array}$ & $\begin{array}{l}0.070 \pm 0.012 \\
(0.056-0.079)\end{array}$ & $\begin{array}{l}<0.01 \pm 0.01 \\
(<0.01-0.01)\end{array}$ & $\begin{array}{l}0.02 \pm 0.00 \\
(0.02-0.02)\end{array}$ & $\begin{array}{l}9.3 \pm 1.2 \\
(8.1-11)\end{array}$ & $\begin{array}{l}0.020 \pm 0.000 \\
(0.019-0.021)\end{array}$ & $\begin{array}{l}0.002 \pm 0.000 \\
(0.002-0.002)\end{array}$ & $\begin{array}{l}0.137 \pm 0.062 \\
(0.089-0.207)\end{array}$ & $\begin{array}{l}0.006 \pm 0.003 \\
(0.003-0.009)\end{array}$ \\
\hline Scylla serrata & 0.080 & 0.061 & 0.001 & 0.071 & 0.01 & 0.03 & 11 & 0.050 & 0.003 & 0.338 & 0.005 \\
\hline Charybdis sp. & $\begin{array}{l}0.079 \pm 0.039 \\
(0.040-0.14)\end{array}$ & $\begin{array}{l}0.030 \pm 0.016 \\
(0.016-0.057)\end{array}$ & $\begin{array}{l}<0.001 \\
(<0.001-0.001)\end{array}$ & $\begin{array}{l}0.055 \pm 0.015 \\
(0.040-0.071)\end{array}$ & $\begin{array}{l}0.02 \pm 0.00 \\
(0.01-0.03)\end{array}$ & $\begin{array}{l}0.03 \pm 0.01 \\
(0.02-0.05)\end{array}$ & $\begin{array}{l}9.2 \pm 0.6 \\
(8.2-10)\end{array}$ & $\begin{array}{l}0.031 \pm 0.010 \\
(0.018-0.040)\end{array}$ & $\begin{array}{l}0.002 \pm 0.001 \\
(0.001-0.003)\end{array}$ & $\begin{array}{l}0.233 \pm 0.084 \\
(0.132-0.341)\end{array}$ & $\begin{array}{l}0.002 \pm 0.001 \\
(0.001-0.003)\end{array}$ \\
\hline Penaeus monodon & $\begin{array}{l}0.16 \pm 0.05 \\
(0.12-0.22)\end{array}$ & $\begin{array}{l}0.044 \pm 0.018 \\
(0.025-0.059)\end{array}$ & $\begin{array}{l}<0.001 \\
<0.001\end{array}$ & $\begin{array}{l}0.072 \pm 0.020 \\
(0.057-0.095)\end{array}$ & $\begin{array}{l}0.01 \pm 0.00 \\
(0.01-0.02)\end{array}$ & $\begin{array}{l}0.03 \pm 0.00 \\
(0.03-0.04)\end{array}$ & $\begin{array}{l}3.4 \pm 0.7 \\
(2.7-4.1)\end{array}$ & $\begin{array}{l}0.032 \pm 0.010 \\
(0.028-0.039)\end{array}$ & $\begin{array}{l}0.001 \pm 0.000 \\
(0.001-0.002)\end{array}$ & $\begin{array}{l}0.125 \pm 0.004 \\
(0.122-0.129)\end{array}$ & $\begin{array}{l}0.001 \pm 0.001 \\
(0.000-0.002)\end{array}$ \\
\hline Penaeus merguiensis & $\begin{array}{l}0.031 \pm 0.025 \\
(0.031-0.025)\end{array}$ & $\begin{array}{l}0.011 \pm 0.002 \\
(0.011-0.002)\end{array}$ & $\begin{array}{l}<0.001 \\
<0.001\end{array}$ & $\begin{array}{l}0.069 \pm 0.024 \\
(0.033-0.085)\end{array}$ & $\begin{array}{l}0.01 \pm 0.01 \\
(<0.01-0.03)\end{array}$ & $\begin{array}{l}0.05 \pm 0.00 \\
(0.04-0.05)\end{array}$ & $\begin{array}{l}3.1 \pm 0.3 \\
(3.1-0.3)\end{array}$ & $\begin{array}{l}0.023 \pm 0.000 \\
(0.023-0.027)\end{array}$ & $\begin{array}{l}0.002 \pm 0.000 \\
(0.002-0.002)\end{array}$ & $\begin{array}{l}0.182 \pm 0.060 \\
(0.144-0.271)\end{array}$ & $\begin{array}{l}0.003 \pm 0.003 \\
(0.001-0.007)\end{array}$ \\
\hline \multicolumn{12}{|l|}{ Cephalopod } \\
\hline Octopus sp. & $\begin{array}{l}0.22 \pm 0.02 \\
(0.20-0.25)\end{array}$ & $\begin{array}{l}0.12 \pm 0.12 \\
(0.046-0.259)\end{array}$ & $\begin{array}{l}<0.001 \\
(<0.001-0.001)\end{array}$ & $\begin{array}{l}0.057 \pm 0.004 \\
(0.052-0.060)\end{array}$ & $\begin{array}{l}0.03 \pm 0.01 \\
(0.02-0.03)\end{array}$ & $\begin{array}{l}0.04 \pm 0.03 \\
(0.02-0.08)\end{array}$ & $\begin{array}{l}0.65 \pm 0.58 \\
(0.29-1.3)\end{array}$ & $\begin{array}{l}0.049 \pm 0.000 \\
(0.047-0.050)\end{array}$ & $\begin{array}{l}0.003 \pm 0.002 \\
(0.001-0.006)\end{array}$ & $\begin{array}{l}0.337 \pm 0.156 \\
(0.210-0.511)\end{array}$ & $\begin{array}{l}0.012 \pm 0.010 \\
(0.004-0.023)\end{array}$ \\
\hline \\
\hline Acanthogobius flavimanus & $\begin{array}{l}0.004 \pm 0.003 \\
(0.001-0.007)\end{array}$ & $\begin{array}{l}0.006 \pm 0.002 \\
(0.004-0.007)\end{array}$ & $\begin{array}{l}0.001 \pm 0.001 \\
(<0.001-0.003)\end{array}$ & $\begin{array}{l}0.054 \pm 0.020 \\
(0.023-0.073)\end{array}$ & $\begin{array}{l}0.02 \pm 0.00 \\
(0.01-0.02)\end{array}$ & $\begin{array}{l}0.04 \pm 0.01 \\
(0.04-0.05)\end{array}$ & $\begin{array}{l}1.6 \pm 0.4 \\
(1.2-2.3)\end{array}$ & $\begin{array}{l}0.037 \pm 0.010 \\
(0.027-0.050)\end{array}$ & $\begin{array}{l}0.002 \pm 0.000 \\
(0.001-0.002)\end{array}$ & $\begin{array}{l}0.371 \pm 0.232 \\
(0.180-0.752)\end{array}$ & $\begin{array}{l}0.005 \pm 0.001 \\
(0.003-0.006)\end{array}$ \\
\hline Buti. & & & . & & $<0.01$ & & 050 & & & & 0.009 \\
\hline Dexillichthys muelleri & & & 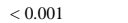 & & 00 & & & & 0.0 & & 0.003 \\
\hline Siganus sp. & $\begin{array}{l}0.003 \\
(0.001-0.004)\end{array}$ & $\begin{array}{l}0.004 \\
(0.002-0.005)\end{array}$ & $\begin{array}{l}<0.001 \\
<0.001\end{array}$ & $\begin{array}{l}0.038 \\
(0.031-0.044)\end{array}$ & $\begin{array}{l}0.01 \\
(0.01-0.01)\end{array}$ & $\begin{array}{l}0.03 \\
(0.02-0.03)\end{array}$ & $\begin{array}{l}0.59 \\
(0.49-0.70)\end{array}$ & $\begin{array}{l}0.018 \\
(0.013-0.022)\end{array}$ & $\begin{array}{l}0.001 \\
(<0.001-0.003)\end{array}$ & $\begin{array}{l}0.084 \\
(0.080-0.089)\end{array}$ & $\begin{array}{l}0.001 \\
(0.001-0.001)\end{array}$ \\
\hline Mugil cephalus & 0.007 & 0.003 & $<0.001$ & 0.052 & 0.01 & 0.05 & 1.7 & 0.024 & 0.004 & 0.268 & 0.006 \\
\hline Plotosus canius & $\begin{array}{l}0.003 \\
(0.003-0.001)\end{array}$ & $\begin{array}{l}0.003 \\
(0.003-0.003)\end{array}$ & $\begin{array}{l}<0.001 \\
(<0.001-0.001)\end{array}$ & $\begin{array}{l}0.037 \\
(0.023-0.051)\end{array}$ & $\begin{array}{l}0.02 \\
(0.01-0.02)\end{array}$ & $\begin{array}{l}0.07 \\
(0.05-0.08)\end{array}$ & $\begin{array}{l}1.5 \\
(1.1-1.9)\end{array}$ & $\begin{array}{l}0.070 \\
(0.060-0.070)\end{array}$ & $\begin{array}{l}0.002 \\
(0.002-0.002)\end{array}$ & $\begin{array}{l}0.218 \\
(0.218-0.119)\end{array}$ & $\begin{array}{l}0.007 \\
(0.007-0.001)\end{array}$ \\
\hline Therapon sp. & $\begin{array}{l}0.001 \\
(0.001-0.002)\end{array}$ & $\begin{array}{l}0.002 \\
(0.001-0.002)\end{array}$ & $\begin{array}{l}<0.001 \\
<0.001\end{array}$ & $\begin{array}{l}0.012 \\
(0.011-0.013)\end{array}$ & $\begin{array}{l}<0.01 \\
<0.01\end{array}$ & $\begin{array}{l}0.03 \\
(0.02-0.03)\end{array}$ & $\begin{array}{l}0.59 \\
(0.59-0.59)\end{array}$ & $\begin{array}{l}0.070 \\
(0.070-0.070)\end{array}$ & $\begin{array}{l}<0.001 \\
<0.001-0.001)\end{array}$ & $\begin{array}{l}0.038 \\
(0.027-0.049)\end{array}$ & $\begin{array}{l}0.003 \\
(0.002-0.003)\end{array}$ \\
\hline Sillago sihama & $\begin{array}{l}0.002 \pm 0.001 \\
(0.002-0.003)\end{array}$ & $\begin{array}{l}0.003 \pm 0.001 \\
(0.002-0.005)\end{array}$ & $\begin{array}{l}<0.001 \\
(<0.001-0.001)\end{array}$ & $\begin{array}{l}0.048 \pm 0.003 \\
(0.044-0.049)\end{array}$ & $\begin{array}{l}0.01 \pm 0.00 \\
(<0.01-0.01)\end{array}$ & $\begin{array}{l}0.04 \pm 0.00 \\
(0.04-0.05)\end{array}$ & $\begin{array}{l}1.2 \pm 0.1 \\
(1.2-1.3)\end{array}$ & $\begin{array}{l}0.028 \pm 0.000 \\
(0.024-0.030)\end{array}$ & $\begin{array}{l}0.001 \pm 0.000 \\
(0.001-0.002)\end{array}$ & $\begin{array}{l}0.256 \pm 0.056 \\
(0.178-0.298)\end{array}$ & $\begin{array}{l}0.002 \pm 0.000 \\
(0.002-0.003)\end{array}$ \\
\hline Oreochromis niloticus & $\begin{array}{l}0.002 \pm 0.000 \\
(0.002-0.002)\end{array}$ & $\begin{array}{l}0.007 \pm 0.001 \\
(0.006-0.008)\end{array}$ & $\begin{array}{l}<0.001 \\
(<0.001-0.001)\end{array}$ & $\begin{array}{l}0.036 \pm 0.005 \\
(0.031-0.040)\end{array}$ & $\begin{array}{l}0.01 \pm 0.00 \\
(0.01-0.01)\end{array}$ & $\begin{array}{l}0.07 \pm 0.03 \\
(0.04-0.11)\end{array}$ & $\begin{array}{l}2.7 \pm 0.8 \\
(1.9-3.6)\end{array}$ & $\begin{array}{l}0.130 \pm 0.020 \\
(0.120-0.170)\end{array}$ & $\begin{array}{l}0.004 \pm 0.003 \\
(0.002-0.007)\end{array}$ & $\begin{array}{l}0.203 \pm 0.093 \\
(0.117-0.325)\end{array}$ & $\begin{array}{l}0.004 \pm 0.002 \\
(0.002-0.006)\end{array}$ \\
\hline Scatophagus argus & $\begin{array}{l}0.003 \pm 0.002 \\
(0.001-0.006)\end{array}$ & $\begin{array}{l}0.003 \pm 0.002 \\
(0.002-0.006)\end{array}$ & $\begin{array}{l}<0.001 \\
(<0.001-0.001)\end{array}$ & $\begin{array}{l}0.030 \pm 0.009 \\
(0.022-0.042)\end{array}$ & $\begin{array}{l}<0.01 \\
(<0.01-0.01)\end{array}$ & $\begin{array}{l}0.06 \pm 0.04 \\
(0.02-0.11)\end{array}$ & $\begin{array}{l}2.3 \pm 1.1 \\
(1.1-3.7)\end{array}$ & $\begin{array}{l}0.033 \pm 0.010 \\
(0.025-0.042)\end{array}$ & $\begin{array}{l}0.007 \pm 0.002 \\
(0.005-0.010)\end{array}$ & $\begin{array}{l}0.276 \pm 0.125 \\
(0.142-0.439)\end{array}$ & $\begin{array}{l}0.003 \pm 0.002 \\
(0.002-0.006)\end{array}$ \\
\hline Ambassis commersoni & $\begin{array}{l}0.003 \\
(0.002-0.004)\end{array}$ & $\begin{array}{l}0.003 \\
(0.002-0.004)\end{array}$ & $\begin{array}{l}<0.001 \\
<0.001\end{array}$ & $\begin{array}{l}0.036 \\
(0.026-0.046)\end{array}$ & $\begin{array}{l}0.01 \\
(0.01-0.01)\end{array}$ & $\begin{array}{l}0.04 \\
(0.04-0.04)\end{array}$ & $\begin{array}{l}1.6 \\
(1.5-1.7)\end{array}$ & $\begin{array}{l}0.054 \\
(0.053-0.055)\end{array}$ & $\begin{array}{l}0.001 \\
(0.001-0.001)\end{array}$ & $\begin{array}{l}0.160 \\
(0.137-0.183)\end{array}$ & $\begin{array}{l}0.003 \\
(0.003-0.003)\end{array}$ \\
\hline Leiogr & 0.007 & 0.003 & $<0.001$ & 0.022 & $<0.01$ & 0.05 & 1.0 & 0.033 & 0.003 & 0.170 & 0.004 \\
\hline Lates calcarifer & $\begin{array}{l}0.002 \pm 0.002 \\
(0.001-0.003)\end{array}$ & $\begin{array}{l}0.001 \pm 0.000 \\
(0.001-0.001)\end{array}$ & $\begin{array}{l}<0.001 \\
<0.001\end{array}$ & $\begin{array}{l}0.013 \pm 0.005 \\
(0.007-0.015)\end{array}$ & $\begin{array}{l}<0.01 \\
<0.01\end{array}$ & $\begin{array}{l}0.04 \pm 0.01 \\
(0.03-0.04)\end{array}$ & $\begin{array}{l}1.6 \pm 1.2 \\
(0.67-2.9)\end{array}$ & $\begin{array}{l}0.080 \pm 0.030 \\
(0.047-0.100)\end{array}$ & $\begin{array}{l}<0.001 \\
(<0.001-0.001)\end{array}$ & $\begin{array}{l}0.022 \pm 0.005 \\
(0.016-0.027)\end{array}$ & $\begin{array}{l}0.004 \pm 0.003 \\
(0.001-0.007)\end{array}$ \\
\hline
\end{tabular}


Am. J. Environ. Sci., 8 (2): 117-129, 2012

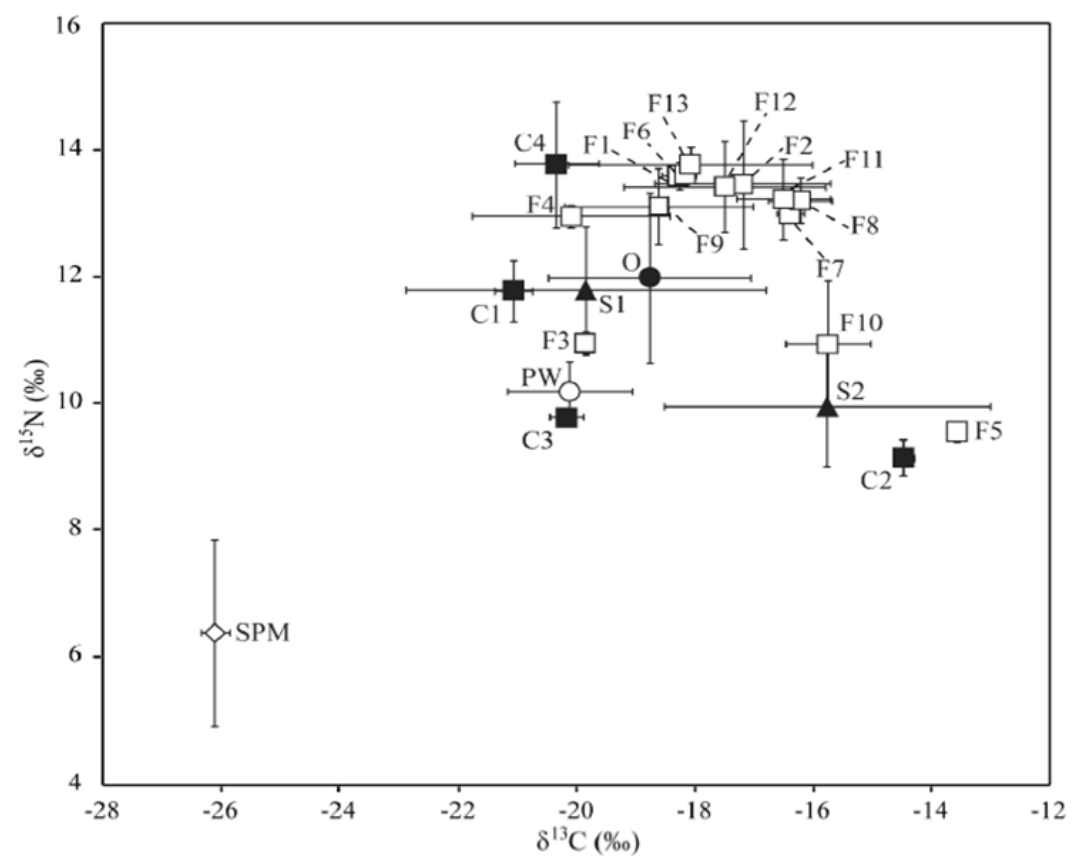

Fig. 2: Relationship between $\delta^{15} \mathrm{~N}$ (mean \pm standard deviation) and $\delta^{13} \mathrm{C}$ (mean \pm standard deviation) values in organisms from an integrated shrimp mangrove farming system, Ba Ria Vung Tau, South Vietnam. See Table 1 for sample sizes. Open diamond, Suspended Particulate Matter (SPM); open square, fish; black square, crab; black triangle, shrimp; open circle, peanut worm Phascolosoma arcuatum (PW); black circle, cephalopod Octopus sp. (O); C1, Calappa sp.; C2, Portunus trituberculatus; C3, Scylla serrata; C4, Charybdis sp.; S1, Penaeus monodon; S2, Penaeus merguiensis; F1, Acanthogobius flavimanus; F2, Butis butis; F3, Dexillichthys muelleri; F4, Siganus sp.; F5, Mugil cephalus; F6, Plotosus canius; F7, Therapon sp.; F8, Sillago sihama; F9, Oreochromis niloticus; F10, Scatophagus argus; F11, Ambassis commersoni; F12, Leiognathus brevirostris; F13, Lates calcarifer

Table 4: Regression statistics for the relationships between trace element concentrations (log-transformed) and $\delta^{15} \mathrm{~N}$ values of biota from the integrated shrimp mangrove farming system, Ba Ria Vung Tau, South Vietnam

\begin{tabular}{|c|c|c|c|c|c|c|c|c|}
\hline \multirow[b]{2}{*}{ Elements } & \multicolumn{4}{|c|}{ Wet weight basis } & \multicolumn{4}{|c|}{ Dry weight basis } \\
\hline & Slope & Intercept & $r$ & $p$-value & Slope & Intercept & $r$ & $p$-value \\
\hline$\overline{\mathrm{V}}$ & -0.080 & 0.169 & -0.357 & 0.007 & -0.085 & 0.770 & -0.371 & 0.005 \\
\hline $\mathrm{Cr}$ & 0.007 & -0.191 & 0.025 & 0.857 & 0.002 & 0.410 & 0.006 & 0.963 \\
\hline $\mathrm{Mn}$ & -0.045 & 1.345 & -0.111 & 0.414 & -0.050 & 1.946 & -0.127 & 0.350 \\
\hline $\mathrm{Fe}$ & -0.110 & 3.120 & -0.502 & 0.000 & -0.115 & 3.721 & -0.509 & 0.000 \\
\hline $\mathrm{Co}$ & -0.141 & 0.824 & -0.505 & 0.000 & -0.146 & 1.425 & -0.520 & 0.000 \\
\hline $\mathrm{Cu}$ & -0.147 & 2.013 & -0.362 & 0.006 & -0.152 & 2.614 & -0.373 & 0.005 \\
\hline $\mathrm{Zn}$ & 0.045 & 0.750 & 0.438 & 0.001 & 0.040 & 1.351 & 0.379 & 0.004 \\
\hline As & 0.007 & -0.161 & 0.040 & 0.772 & 0.002 & 0.440 & 0.010 & 0.939 \\
\hline $\mathrm{Se}$ & 0.034 & -0.877 & 0.342 & 0.010 & 0.029 & -0.276 & 0.276 & 0.039 \\
\hline $\mathrm{Rb}$ & -0.028 & 0.236 & -0.443 & 0.001 & $-\mathbf{0 . 0 3 3}$ & 0.837 & -0.440 & 0.001 \\
\hline $\mathrm{Sr}$ & 0.007 & 1.935 & 0.021 & 0.877 & 0.002 & 2.537 & 0.007 & 0.960 \\
\hline Mo & -0.109 & 0.071 & -0.488 & 0.000 & -0.114 & 0.672 & -0.489 & 0.000 \\
\hline $\mathrm{Ag}$ & -0.159 & -0.562 & -0.367 & 0.005 & -0.164 & 0.039 & -0.378 & 0.004 \\
\hline $\mathrm{Cd}$ & -0.123 & -1.103 & -0.381 & 0.004 & -0.128 & -0.502 & -0.388 & 0.003 \\
\hline In & -0.052 & -3.350 & -0.159 & 0.241 & -0.058 & -2.749 & -0.173 & 0.202 \\
\hline $\mathrm{Sn}$ & $-\mathbf{0 . 0 7 0}$ & -1.032 & -0.471 & 0.000 & -0.075 & -0.431 & -0.481 & 0.000 \\
\hline $\mathrm{Sb}$ & -0.015 & -2.333 & -0.090 & 0.509 & -0.020 & -1.732 & -0.116 & 0.396 \\
\hline Cs & -0.036 & -1.457 & -0.272 & 0.043 & -0.041 & -0.856 & -0.289 & 0.031 \\
\hline $\mathrm{Ba}$ & -0.085 & 0.868 & -0.343 & 0.010 & -0.090 & 1.469 & -0.381 & 0.004 \\
\hline $\mathrm{Hg}$ & 0.053 & -2.599 & 0.387 & 0.003 & 0.048 & -1.998 & 0.356 & 0.007 \\
\hline $\mathrm{Tl}$ & -0.112 & -1.890 & -0.489 & 0.000 & $-\mathbf{0 . 1 1 7}$ & -1.289 & -0.512 & 0.000 \\
\hline $\mathrm{Pb}$ & -0.090 & -0.150 & -0.380 & 0.004 & -0.095 & 0.451 & -0.381 & 0.004 \\
\hline $\mathrm{Bi}$ & -0.080 & -2.062 & -0.301 & 0.024 & -0.085 & -1.461 & -0.306 & 0.022 \\
\hline
\end{tabular}

Bold number for slope indicate significance at $p<0.05$ 

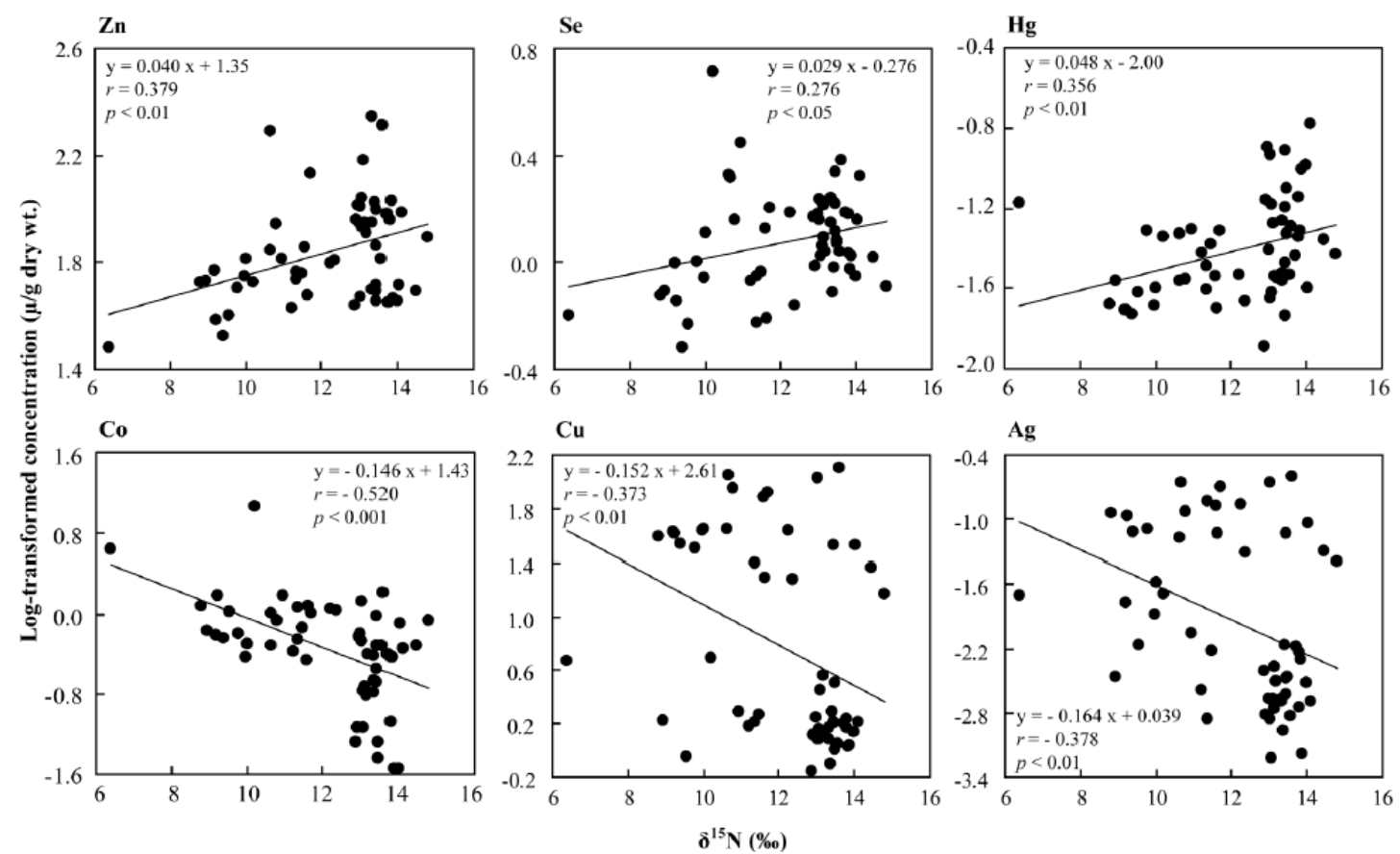

Fig. 3: Relationships between $\delta^{15} \mathrm{~N}$ and selected log-transformed trace element concentrations ( $\mu \mathrm{g} / \mathrm{g}$ dry wt.) in biota from the integrated shrimp mangrove farming system, Ba Ria Vung Tau, South Vietnam

Table 5: Molar Se:Hg ratios (mean \pm standard deviation) of biota from the integrated shrimp mangrove farming system, Ba Ria Vung Tau, South Vietnam

\begin{tabular}{|c|c|c|c|}
\hline Species & Se: $\mathrm{Hg}$ & Species & Se:Hg \\
\hline SPM & 24 & Fish & \\
\hline Sipuncula & & Acanthogobius flavimanus & $76 \pm 23$ \\
\hline Phascolosoma arcuatum & 288 & Butis butis & 39 \\
\hline Crustacean & & Dexillichthys muelleri & 144 \\
\hline Calappa sp. & $76 \pm 6$ & Siganus sp. & $227 \pm 90$ \\
\hline Portunus trituberculatus & $84 \pm 6$ & Mugil cephalus & 62 \\
\hline Scylla serrata & 52 & Plotosus canius & $60 \pm 8$ \\
\hline Charybdis sp. & $114 \pm 55$ & Therapon sp. & $38 \pm 4$ \\
\hline Penaeus monodon & $120 \pm 10$ & Sillago sihama & $112 \pm 11$ \\
\hline Penaeus merguiensis & $141 \pm 40$ & Oreochromis niloticus & $36 \pm 7$ \\
\hline Cephalopod & & Scatophagus argus & $62 \pm 7$ \\
\hline \multirow[t]{3}{*}{ Octopus sp. } & $105 \pm 19$ & Ambassis commersoni & $79 \pm 1$ \\
\hline & & Leiognathus brevirostris & 73 \\
\hline & & Lates calcarifer & $37 \pm 23$ \\
\hline
\end{tabular}

Significant positive linear relationships were found between $\log$ molar concentrations of $\mathrm{Se}$ and $\mathrm{Hg}$ for fishes $(\log$ molar $\mathrm{Se}=-0.927+0.245[\log$ molar $\mathrm{Hg}], r$ $=0.422, p<0.05)$ and all organisms $(\log$ molar $\mathrm{Se}=-$ $0.777+0.282$ [log molar Hg], $r=0.368, p<0.01)$.

\section{DISCUSSION}

Food web structure of ISMFS: In the present study, the $\delta^{15} \mathrm{~N}$ measurements showed a stable enrichment with increasing relative trophic position (Fig. 2) from SPM to swimming crab Charybdis sp. and barramundi seabass Lates calcarifer at the top of the ISMFS trophic web. According to Hobson and Welch (1992), an estimate of the trophic enrichment factor for nitrogen can be obtained by examining isotopic differences between consumers and their diets in cases where such a trophic relationship is well established. Young barramundi seabass Lates calcarifer $(30-50 \mathrm{~cm}$ total length) feed mostly on juvenile Penaeus merguiensis (Robertson and Duke, 1987; Robertson, 1988; Salini et al., 1990); hence, a mean trophic enrichment factor of approximately $4.0 \%$ occurred between these two species in this study. The trophic difference of $\delta 1^{5} \mathrm{~N}$ between the peanut worm Phascolosoma arcuatum and its primary food (SPM) were also about $4.0 \%$. Thus, this trophic enrichment factor for $\delta^{15} \mathrm{~N}$ was likely applicable throughout the ISMFS food web. These results showed that the food web of this ISMFS analyzed had three trophic levels.

In our study isotopic carbon composition of sediment (-25.4\%o) was comparable to that of SPM ($26.1 \%$ ). These $\delta^{13} \mathrm{C}$ values are close to those of mangrove leaves and benthic detritus reported for mangrove ecosystems in Malaysia, Thailand and Indonesia (Rodelli et al., 1984; Thimdee et al., 2004; 2008; Herbon, 2011), indicating mangrove leaves as one of carbon sources in this ISMFS. In contrast with 
$\delta^{15} \mathrm{~N}$, Phascolosoma arcuatum was $6 \%$ enriched in ${ }^{13} \mathrm{C}$ versus SPM, while Lates calcarifer was $2.3 \%$ o depleted in $\delta^{13} \mathrm{C}$ versus Penaeus merguiensis. Mean $\delta^{13} \mathrm{C}$ for benthic microalgae in mangrove ecosystems was reported between $-19.3 \%$ (Hsieh et al., 2002) and 17.3\%o (Bouillon et al., 2002). Phascolosoma arcuatum occupies the second trophic level with intermediate $\delta^{13} \mathrm{C}(-20.1 \%$ o), suggesting that this species probably feed on mangrove and benthic microalgae -derived carbon sources as found in Malaysian and Indonesian mangrove ecosystems (Rodelli et al., 1984; Herbon, 2011). Lates calcarifer occurs on the third trophic level with $\delta^{13} \mathrm{C}$ of $-18.1 \%$, indicating this species is a carnivore eating a wide range of prey. As stated by Peterson and Fry (1987), intermediate isotopic value is a common problem of isotopic tracer studies when more than two sources are present.

Trace element concentrations in the sediment and biota: The concentrations of trace elements in these two sediments from the ISMFS were lower than those collected from the Mekong River Delta and Sai GonDong Nai River (Phuong et al., 1998; Anh et al., 2003; Cenci and Martin, 2004), indicating that there is little pollution in this mangrove ecosystem.

High accumulations of $\mathrm{Cu}, \mathrm{Zn}, \mathrm{Ag}$ and $\mathrm{Cd}$ in cephalopods have been shown in previous studies (e.g., Bustamante et al., 1998a; 1998b; Ichihashi et al., 2001). Copper is known to be essential to marine mollusks. Across mollusk taxa, $\mathrm{Cd}$ and $\mathrm{Cu}$ generally bind to the same type of metal binding proteins, e.g., metallothioneins, in the digestive gland (Bustamante et al., 1998b); but there is as yet no clear explanation for the high accumulation of $\mathrm{Ag}$ in cephalopods (Ichihashi et al., 2001). Manganese, $\mathrm{Sr}$ and Ba generally appeared to be the most highly concentrated elements in the crustaceans we analyzed. Due to their chemical similarity to calcium, Mn, $\mathrm{Sr}$ and $\mathrm{Ba}$ are readily accumulated in the exoskeletons of crustaceans (Paez-Osuna et al., 1995; Pourang et al., 2004). Ikemoto et al. (2008) reported high Mn, As and Ba in crustaceans from the Mekong River Delta, South Vietnam. Our previous studies on black tiger shrimp and giant river prawn from South Vietnam also found a high accumulation of $\mathrm{Mn}$ and $\mathrm{Ba}$ in the exoskeletons (Tu et al., 2008a; 2008b).

High accumulation of $\mathrm{Hg}$ in fish has been widely demonstrated in earlier studies (Prudente et al., 1997; Agusa et al., 2005; Ikemoto et al., 2008). In this study, $\mathrm{Hg}$ concentration was comparatively high in tilapia Oreochromis niloticus samples analyzed, ranging from $0.120-0.170 \mu \mathrm{g} \mathrm{g}^{-1}$ dry wt. As reported by Benson et al. (2007), tilapia collected from tropical aquatic ecosystems in the Niger Delta, Nigeria accumulated relatively high of $\mathrm{Hg}$.
Observed concentrations of trace elements in the ISMFS organisms in the present study were comparable to or lower than those reported elsewhere (Prudente et al., 1997; Bustamante et al., 1998a; 1998b; Ichihashi et al., 2001; Agusa et al., 2005; Ikemoto et al., 2008). Food guideline values for $\mathrm{Cu}\left(20 \mu \mathrm{g} \mathrm{g}^{-1}\right.$ wet weight (wet wt.)), $\mathrm{Zn}\left(50 \mu \mathrm{g} \mathrm{g}^{-1}\right.$ wet wt.), Cd (0.2 $\mu \mathrm{g} \mathrm{g}^{-1}$ wet wt.), $\mathrm{Hg}(0.3 \mu \mathrm{g}$ $\mathrm{g}^{-1}$ wet wt.) and $\mathrm{Pb}\left(2 \mu \mathrm{g} \mathrm{g}^{-1}\right.$ wet wt. for fish and $10 \mu \mathrm{g} \mathrm{g}^{-1}$ wet wt. for shellfish), in fish and shellfish have been summarized by the Ministry of Agriculture, Fisheries and Food (MAFF) in the UK (MAFF, 2000). In the present study, the concentrations of toxic metals $(\mathrm{Cd}, \mathrm{Hg}$ and $\mathrm{Pb})$ in all fish and shellfish samples from the ISMFS, BRVT were below the MAFF standards, however, black tiger shrimps (3/3 samples) and octopus (2/3 samples) had concentrations of $\mathrm{Cu}$ that exceeded the MAFF standards. Our previous report also demonstrated a high concentration of $\mathrm{Cu}$ in black tiger shrimp in South Vietnam (Tu et al., 2008b). Further, the concentration of $\mathrm{Zn}$ in one sample of Commerson's glassy fish Ambassis commersoni (65 $\mu \mathrm{g} \mathrm{g}^{-1}$ wet wt.) was higher than the MAFF guideline.

Trophic transfer of trace elements: Similar relationships between $\mathrm{Hg}$ concentration and $\delta^{15} \mathrm{~N}$ have been found in other aquatic food webs (Atwell et al., 1998; Bowles et al., 2001; Campbell et al., 2005; AlReasi et al., 2007; Ikemoto et al., 2008). The log $\mathrm{Hg}$ concentration- $\delta^{15} \mathrm{~N}$ regression slope of 0.048 found in this study was comparable to the slope of 0.07 reported for $\mathrm{Hg}$ in a tropical coastal food web in the Gulf of Oman (Al-Reasi et al., 2007). However, this slope was remarkably smaller than that observed for the Mekong River food web (0.114) (Ikemoto et al., 2008), Arctic marine food webs (0.197-0.32) (Jarman et al., 1996; Atwell et al., 1998; Campbell et al., 2005) and for biota in Lake Murray, Papua New Guinea (0.28) (Bowles et al., 2001). According to Al-Reasi et al. (2007), lower biomagnification in tropical marine food webs could be due to a diversity of diet items with different $\mathrm{Hg}$ concentrations that result in similar $\delta^{15} \mathrm{~N}$ for each species but highly variable $\mathrm{Hg}$ burden. Furthermore, the differences between the slopes of the $\log \mathrm{Hg}$ concentration- $\delta \delta^{15} \mathrm{~N}$ regression may reflect the differences in the food web structure (e.g., number of trophic levels) and/or differences in growth rate (Ikemoto et al., 2008). Atwell et al. (1998) and Campbell et al. (2005) measured Hg concentrations in marine mammals and seabirds, which are known to specifically accumulate large amounts of $\mathrm{Hg}$ in their tissues. In general, organisms in tropical areas have faster growth rates, which may lead to lower biomagnification of $\mathrm{Hg}$ even at high trophic levels (Ikemoto et al., 2008). 
Zinc and Se were also found to biomagnify in this ISMFS food web. The slopes of the log concentration$\delta^{15} \mathrm{~N}$ regressions were 0.040 for $\mathrm{Zn}$ and 0.029 for Se in the present study, suggesting that these elements biomagnify less than Hg. Stewart et al. (2004) found significant positive correlations between $\mathrm{Se}$ concentrations and $\delta^{15} \mathrm{~N}$ in San Francisco Bay. Moreover, trophic transfer of Se was observed between trophic levels from prey (microplankton and mesoplankton) to top predator (fish) in the Guanabara Bay food web in the western South Atlantic (Kehrig et al., 2009a). Zinc also biomagnified in pelagic Arctic marine and Montana stream invertebrate food webs (Quinn et al., 2003; Campbell et al., 2005).

According to Campbell et al. (2005), biodiminution is a decrease in the concentration of an element with increasing trophic level, i.e., a significant negative slope between log element concentration and $\delta^{15} \mathrm{~N}$. Biodiminution of a number of elements was observed in the ISMFS food web. Concentrations of metals decreases at a higher trophic level in the marine planktonic food chain is mainly the result of an effective efflux of metals from copepods and a very low assimilation of metals by marine fish (Wang, 2002).

In several studies, the ratio between $\mathrm{Hg}$ and $\mathrm{Se}$ molar concentrations was close to equimolarity in marine mammals and birds, but a large excess of Se in relation to $\mathrm{Hg}$ was observed in fishes and crustaceans (Andersen and Depledge, 1997; Dietz et al., 2000; Ikemoto et al., 2004; Kunito et al., 2004; Campbell et al., 2005; Kehrig et al., 2009b). It has been shown that various vertebrates are able to detoxify $\mathrm{Hg}$ by a specific chemical mechanism involving Se, suggesting that Se has an antagonistic effect on $\mathrm{Hg}$ metabolism (Elia et al., 2003; Ikemoto et al., 2004; Kunito et al., 2004). This relation has also been documented in marine ecosystem (Dietz et al., 2000; Campbell et al., 2005; Kehrig et al., 2009b). Compared to our study, the mean $\mathrm{Se} / \mathrm{Hg}$ molar ratio $(56.4 \pm 47.5)$ of whole crustacean in Greenland marine environment (Dietz et al., 2000) was comparable to Calappa sp., Portunus trituberculatus and Scylla serrata, but lower than that of Charybdis sp., Penaeus monodon and Penaeus merguiensis. Most of $\mathrm{Se} / \mathrm{Hg}$ molar ratios of fish in the ISMFS food web were higher than those of fish in Greenland water (Dietz et al., 2000) and Guanabara Bay, Brazil (Kehrig et al., 2009b). Chen et al. (2001) found that the presence of Se in fish muscles was positively correlated to the concentrations of Se in waters and that the presence of Se in fish muscles appeared to be an explanation for the limited accumulation of $\mathrm{Hg}$ in fish. Despite the potential $\mathrm{Se} / \mathrm{Hg}$ antagonism in organisms, great care should be taken when using Se as a remediating agent against $\mathrm{Hg}$, because paradox of Se (nutritionally required in small amounts but highly toxic in slightly greater amounts) (Lemly, 1993). Lemly (1993) showed that food-chain organisms containing $3 \mu \mathrm{g} \mathrm{Se} / \mathrm{g}$ dry wt. or more should be viewed as potentially lethal to fish and aquatic birds that consume them. Fortunately, Se concentrations in most of organisms collected from the ISMFS food web were less than this threshold, except the peanut worm Phascolosoma arcuatum $\left(5.2 \mu \mathrm{g} \mathrm{g}^{-1}\right)$.

\section{CONCLUSION}

The results of this study highlight the usefulness of $\delta^{13} \mathrm{C}$ and $\delta^{15} \mathrm{~N}$ as a tool not only for elucidating the trophic position of biota, but also for tracing contaminants within food webs.

\section{ACKNOWLEDGEMENT}

This study was partially supported by grants from the Global COE Program from the Ministry of Education, Culture, Sports, Science and Technology, the Research Grant-in-Aid for Scientific Research from the Japanese Society for the Promotion of Science (JSPS) (Nos. 19310024, 20221003) and Grant-in-Aid for JSPS Fellow (No. 2109237 to NPCT).

\section{REFERENCES}

Agusa, T., T. Kunito, G. Yasunaga, H. Iwata and A. Subramanian et al., 2005. Concentrations of trace elements in marine fish and its risk assessment in Malaysia. Mar. Pollut. Bull., 51: 896-911. DOI: 10.1016/j.marpolbul.2005.06.007.

Agusa, T., S. Inoue, T. Kunito, T.B. Minh and N.N. Ha et al., 2009. Human exposure to arsenic from groundwater in the Red River and Mekong River Deltas in Vietnam. Int. J. Environ. Stud., 66: 49-57. DOI: $10.1080 / 00207230902759962$

Al-Reasi, H.A., F.A. Ababneh and D.R. Lean, 2007. Evaluating mercury biomagnification in fish from a tropical marine environment using stable isotopes $\left({ }^{13} \mathrm{C}\right.$ and $\left.\delta^{15} \mathrm{~N}\right)$. Environ. Toxicol. Chem., 26: 1572-1581. DOI: $10.1897 / 06-359 R .1$

Andersen, J.L. and M.H. Depledge, 1997. A survey of total mercury and methylmercury in edible fish and invertebrates from Azorean waters. Mar. Environ. Res., 44: 331-350. DOI: 10.1016/S01411136(97)00011-1

Anh, M.T., L.C.D. Hong, N.V. Nguyen, C.L.T. Thi, T.L. Minh, K.B.-V. Slooten and J. Tarradellas, 2003. Micropollutants in the sediment of the Saigon-Dongnai river: Situation and ecological risks. Chimia, 57: 537-541.

Atwell, L., K.A. Hobson and H.E. Welch, 1998. Biomagnification and bioaccumulation of mercury in an arctic marine food web: Insights from stable nitrogen isotope analysis. Can. J. Fish. Aquat. Sci., 55: 1114-1121. DOI: 10.1139/f98-001 
Benson, N.U., J.P. Essien, A.B. Williams and D.E. Bassey, 2007. Mercury accumulation in fishes from tropical aquatic ecosystems in the Niger Delta, Nigeria. Curr. Sci., 92: 781-785. http://cstest.ias.ac.in/cs/Downloads/article_41105.pdf

Binh, C.T., M.J. Phillips and H. Demaine, 1997. Integrated shrimp-mangrove farming systems in the Mekong delta of Vietnam. Aquac. Res., 28: 599-610. DOI: 10.1046/j.1365-2109.1997.00901.x

Bouillon, S., N. Koedam, A.V. Raman and F. Dehairs, 2002. Primary producers sustaining macroinvertebrate communities in intertidal mangrove forests. Oecologia, 130: 441-448. DOI: 10.1007/s004420100814

Bowles, K.C., S.C. Apte, W.A. Maher, M. Kawei and R. Smith, 2001. Bioaccumulation and biomagnification of mercury in Lake Murray, Papua New Guinea. Can. J. Fish. Aquat. Sci., 58: 888-897. DOI: 10.1139/f01-042

Bustamante, P., F. Caurant, S.W. Fowler and P. Miramand, 1998a. Cephalopods as a vector for the transfer of cadmium to top marine predators in the north-east Atlantic Ocean. Sci. Total Environ., 220: 71-80. DOI: 10.1016/S0048-9697(98)00250-2

Bustamante, P., Y. Cherel, F. Caurant and P. Miramand, 1998b. Cadmium, copper and zinc in octopuses from Kerguelen Islands, Southern Indian Ocean. Polar Biol., 19: 264-271. DOI: 10.1007/s003000050244

Cabana, G. and J.B. Rasmussen, 1994. Modelling food chain structure and contaminant bioaccumulation using stable nitrogen isotopes. Nature, 372: 255257. DOI: $10.1038 / 372255 \mathrm{a} 0$

Campbell, L.M., R.J. Norstrom, K.A. Hobson, D.C.G. Muir, S. Backus and A.T. Fisk, 2005. Mercury and other trace elements in a pelagic Arctic marine food web (Northwater Polynya, Baffin Bay). Sci. Total Environ., 351-352: 247-263. DOI: 10.1016/j.scitotenv.2005.02.043

Cenci, R.M. and J.M. Martin, 2004. Concentration and fate of trace metals in Mekong River Delta. Sci. Total Environ., 332: 167-182. DOI: 10.1016/j.scitotenv.2004.01.018

Chen, Y.W., N. Belzile and J.M. Gunn, 2001. Antagonistic effect of selenium on mercury assimilation by fish populations near Sudbury metal smelters? Limnol. Oceanogr., 46: 1814-1818. DOI: 10.4319/lo.2001.46.7.1814

Costanzo, S.D., M.J. O'Donohue and W.C. Dennison, 2004. Assessing the influence and distribution of shrimp pond effluent in a tidal mangrove creek in north-east Australia. Mar. Pollut. Bull., 48: 514525. DOI: 10.1016/j.marpolbul.2003.09.006
Costanzo, S.D., J. Udy, B. Longstaff and A. Jones, 2005. Using nitrogen stable isotope ratios $\left(\delta^{15} \mathrm{~N}\right)$ of macroalgae to determine the effectiveness of sewage upgrades: changes in the extent of sewage plumes over four years in Moreton Bay, Australia. Mar. Pollut. Bull., 51: 212-217. DOI: 10.1016/j.marpolbul.2004.10.018

DeNiro, M.J. and S. Epstein, 1978. Influence of diet on the distribution of carbon isotopes in animals. Geochim. Cosmochim. Acta, 42: 495-506. DOI: 10.1016/0016-7037(78)90199-0

Dietz, R., F. Riget and E.W. Born, 2000. An assessment of selenium to mercury in Greenland marine animals. Sci. Total Environ., 245: 15-24. DOI: 10.1016/S0048-9697(99)00430-1

Elia, A.C., R. Galarini, M.I. Taticchi, A.J.M. Dörr and L. Mantilacci, 2003. Antioxidant responses and bioaccumulation in Ictalurus melas under mercury exposure. Ecotoxicol. Environ. Saf., 55: 162-167. DOI: 10.1016/S0147-6513(02)00123-9

Herbon, C.M., 2011. Spatial and temporal variability in benthic food webs of the mangrove fringed Segara Anakan Lagoon in Java, Indonesia. Ph.D thesis, University of Bremen, Bremen, Germany. http://elib.suub.unibremen.de/edocs/001019361.pdf

Hobson, K.A. and H.E. Welch, 1992. Determination of trophic relationships within a high Arctic marine food web using $\delta^{13} \mathrm{C}$ and $\delta^{15} \mathrm{~N}$ analysis. Mar. Ecol. Prog. Ser., 84: 9-18.

Hsieh, H.L., C.P. Chen, Y.G. Chen and H.H. Yang, 2002. Diversity of benthic organic matter flows through polychaetes and crabs in a mangrove estuary: $\delta^{13} \mathrm{C}$ and $\delta^{34} \mathrm{~S}$ signals. Mar. Ecol. Prog. Ser., 227: 145-155. DOI: 10.3354/meps227145

Ichihashi, H., H. Kohno, K. Kannan, A. Tsumura and S.I. Yamasaki, 2001. Multielemental analysis of purpleback flying squid using high resolution inductively coupled plasma-mass spectrometry (HR ICP-MS). Environ. Sci. Technol., 35: 31033108. DOI: 10.1021/es010653v

Ikemoto, T., T. Kunito, Y. Anan, H. Tanaka and N. Baba et al., 2004. Association of heavy metals with metallothionein and other proteins in hepatic cytosol of marine mammals and seabirds. Environ. Toxicol. Chem., 23: 2008-2016. DOI: 10.1897/03-456.

Ikemoto, T., N.P.C. Tu, N. Okuda, A. Iwata and K. Omori et al., 2008. Biomagnification of trace elements in the aquatic food web in the Mekong Delta, South Vietnam using stable carbon and nitrogen isotope analysis. Arch. Environ. Contam. Toxicol., 54: 504-515. DOI: 10.1007/s00244-0079058-5 
Jarman, W.M., K.A. Hobson, W.J. Sydeman, C.E. Bacon and E.B. McLaren, 1996. Influence of trophic position and feeding location on contaminant levels in the Gulf of the Farallones food web revealed by stable isotope analysis. Environ. Sci. Technol., 30: 654-660. DOI: 10.1021/es950392n

Jones, A.B., M.J. O'Donohue, J. Udy and W.C. Dennison, 2001. Assessing ecological impacts of shrimp and sewage effluent: Biological indicators with standard water quality analyses. Estuar. Coast. Shelf Sci., 52: 91-109. DOI: 10.1006/ecss.2000.0729

Kehrig, H.A., E.F.A. Palermo, T.G. Seixas, C.W.C. Branco, I. Moreira and O. Malm, 2009a. Trophic transfer of methylmercury and trace elements by tropical estuarine seston and plankton. Estuar. Coast. Shelf Sci., 85: 36-44. DOI: 10.1016/j.ecss.2009.05.027

Kehrig, H.A., T.G. Seixas, E.A. Palermo, A.P. Baeta and C.W.C. Branco et al., 2009b. The relationships between mercury and selenium in plankton and fish from a tropical food web. Environ. Sci. Pollut. Res., 16: 10-24. DOI: 10.1007/s11356-008-0038-8

Kidd, K.A., R.H. Hesslein, R.J.P. Fudge and K.A. Hallard, 1995. The influence of trophic level as measured by $\delta^{15} \mathrm{~N}$ on mercury concentrations in freshwater organisms. Water, Air, Soil Pollut., 80: 1011-1015. DOI: 10.1007/BF01189756

Kunito, T., S. Nakamura, T. Ikemoto, Y. Anan and R. Kubota et al., 2004. Concentration and subcellular distribution of trace elements in liver of small cetaceans incidentally caught along the Brazilian coast. Mar. Pollut. Bull., 49: 574-587. DOI: 10.1016/j.marpolbul.2004.03.009

Lemly, A.D., 1993. Guidelines for evaluating selenium data from aquatic monitoring and assessment studies. Environ. Monit. Assess., 28: 83-100. DOI: 10.1007/bf00547213

MAFF, 2000. Monitoring and surveillance of nonradioactive contaminants in the aquatic environment and activities regulating the disposal of wastes at sea, 1997. Ministry of Agriculture, Fisheries and Food of United Kingdom, Aquatic Environment Monitoring Report.

Minagawa, M. and E. Wada, 1984. Stepwise enrichment of ${ }^{15} \mathrm{~N}$ along food chains: Further evidence and the relation between $\delta^{15} \mathrm{~N}$ and animal age. Geochim. Cosmochim. Acta, 48: 1135-1140. DOI: 10.1016/0016-7037(84)90204-7

MONRE, 2004. Country report on land based pollution in Vietnam 2004. Vietnamese Ministry of Natural Resources and Environment, Hanoi.
Paez-Osuna, F., R. Perez-Gonzalez, G. Izaguirre-Fierro, H.M. Zazueta-Padilla and L.M. Flores-Campaña, 1995. Trace metal concentrations and their distribution in the lobster Panulirus inflatus (Bouvier, 1895) from the Mexican Pacific coast. Environ. Pollut., 90: 163-170. DOI: 10.1016/02697491(94)00103-K

Peterson, B.J. and B. Fry, 1987. Stable isotopes in ecosystem studies. Annu. Rev. Ecol. Syst., 18: 293320. DOI: 10.1146/annurev.es.18.110187.001453

Phuong, P.K., C.P.N. Son, J.J. Sauvain and J. Tarradellas, 1998. Contamination by PCB's, DDT's and heavy metals in sediments of Ho Chi Minh City's canals, Vietnam. Bull. Environ. Contam. Toxicol., 60: 347-354. DOI: 10.1007/s001289900633

Pourang, N., J.H. Dennis and H. Ghourchian, 2004. Tissue distribution and redistribution of trace elements in shrimp species with the emphasis on the roles of metallothionein. Ecotoxicology, 13: 519-533.

DOI: 10.1023/B:ECTX.0000037189.80775.9c

Prudente, M., E.Y. Kim, S. Tanabe and R. Tatsukawa, 1997. Metal levels in some commercial fish species from Manila Bay, the Philippines. Mar. Pollut. Bull., 34: 671-674. DOI: 10.1016/S0025326X(97)00035-0

Quinn, M.R., X. Feng, C.L. Folt and C.P. Chamberlain, 2003. Analyzing trophic transfer of metals in stream food webs using nitrogen isotopes. Sci. Total Environ., 317: 73-89. DOI: 10.1016/S00489697(02)00615-0

Robertson, A.I. and N.C. Duke, 1987. Mangroves as nursery sites: comparisons of the abundance and species composition of fish and crustaceans in mangroves and other nearshore habitats in tropical Australia. Mar. Biol., 96: 193-205. DOI: 10.1007/BF00427019

Robertson, A.I., 1988. Abundance, diet and predators of juvenile banana prawns, Penaeus merguiensis, in a tropical mangrove estuary. Mar. Freshw. Res., 39: 467-478. DOI: 10.1071/MF9880467

Rodelli, M.R., J.N. Gearing, P.J. Gearing, N. Marshall and A. Sasekumar, 1984. Stable isotope ratio as a tracer of mangrove carbon in Malaysian ecosystems. Oecologia, 61: 326-333. DOI: 10.1007/BF00379629

Salini, J.P., S.J.M. Blaber and D.T. Brewer, 1990. Diets of piscivorous fishes in a tropical Australian estuary, with special reference to predation on penaeid prawns. Mar. Biol., 105: 363-374. DOI: 10.1007/BF01316307 
Stewart, A.R., S.N. Luoma, C.E. Schlekat, M.A. Doblin and K.A. Hieb, 2004. Food web pathway determines how selenium affects aquatic ecosystems: a San Francisco Bay case study. Environ. Sci. Technol., 38: 4519-4526. DOI: 10.1021/es0499647

Suedel, B.C., J.A. Boraczek, R.K. Peddicord, P.A. Clifford and T.M. Dillon, 1994. Trophic transfer and biomagnification potential of contaminants in aquatic ecosystems. Rev. Environ. Contam. Toxicol., 136: 21-89. PMID: /8029491

Thimdee, W., G. Deein, C. Sangrungruang and K. Matsunaga, 2004. Analysis of primary food sources and trophic relationships of aquatic animals in a mangrove-fringed estuary, Khung Krabaen Bay (Thailand) using dual stable isotope techniques. Wetl. Ecol. Manag., 12: 135-144. DOI: 10.1023/B:WETL.0000021674.76171.69

Thimdee, W., G. Deein, N. Nakayama, Y. Suzuki and K. Matsunaga, 2008. $\delta^{13} \mathrm{C}$ and $\delta^{15} \mathrm{~N}$ indicators of fish and shrimp community diet and trophic structure in a mangrove ecosystem in Thailand. Wetl. Ecol. Manag., 16: 463-470. DOI: 10.1007/s11273-008-9083-9
Tu, N.P.C., N.N. Ha, T. Ikemoto, B.C. Tuyen, S. Tanabe and I. Takeuchi, 2008a. Bioaccumulation and distribution of trace elements in tissues of giant river prawn Macrobrachium rosenbergii (Decapoda: Palaemonidae) from South Vietnam. Fish. Sci., 74: 109-119. DOI: 10.1111/j.14442906.2007.01474.x

Tu, N.P.C., N.N. Ha, T. Ikemoto, B.C. Tuyen, S. Tanabe and I. Takeuchi, 2008b. Regional variations in trace element concentrations in tissues of black tiger shrimp Penaeus monodon (Decapoda: Penaeidae) from South Vietnam. Mar. Pollut. Bull., 57: 858-866. DOI: 10.1016/j.marpolbul.2008.02.016.

Tu, N.P.C., T. Agusa, N.N. Ha, B.C. Tuyen, S. Tanabe and I. Takeuchi, 2011. Stable isotope-guided analysis of biomagnification profiles of arsenic species in a tropical mangrove ecosystem. Mar. Pollut. Bull., 63: 124-134. DOI: 10.1016/j.marpolbul.2011.03.002

Wang, W.X., 2002. Interactions of trace metals and different marine food chains. Mar. Ecol. Prog. Ser., 243: 295-309. DOI: 10.3354/meps243295 